\title{
Novel Iterative Neural Dynamic Programming for Data-Based Approximate Optimal Control Design *
}

\author{
Chaoxu Mu ${ }^{\mathrm{a}}$, Ding Wang ${ }^{\mathrm{b}}$, Haibo $\mathrm{He}^{\mathrm{c} *}$ \\ ${ }^{a}$ Tianjin Key Laboratory of Process Measurement and Control, School of Electrical and Information Engineering, Tianjin \\ University, Tianjin 300072, China \\ ${ }^{\mathrm{b}}$ The State Key Laboratory of Management and Control for Complex Systems, Institute of Automation, Chinese Academy of \\ Sciences, Beijing 100190, China \\ ${ }^{\mathrm{c}}$ Department of Electrical, Computer and Biomedical Engineering, University of Rhode Island, RI, USA
}

\begin{abstract}
As a powerful method of solving the nonlinear optimal control problem, the iterative adaptive dynamic programming (IADP) is usually established on the known controlled system model and is particular for affine nonlinear systems. Since most nonlinear systems are complicated to establish accurate mathematical models, this paper provides a novel data-based approximate optimal control algorithm, named iterative neural dynamic programming (INDP) for affine and non-affine nonlinear systems by using system data rather than accurate system models. The INDP strategy is built within the framework of IADP, where the convergence guarantee of the iteration is provided. The INDP algorithm is implemented based on the model-based heuristic dynamic programming (HDP) structure, where model, action and critic neural networks are employed to approximate the system dynamics, the control law and the iterative cost function, respectively. During the back-propagation of action and critic networks, the approach of directly minimizing the iterative cost function is developed to eliminate the requirement of establishing system models. The neural network implementation of the INDP algorithm is presented in details and the associated stability is also analyzed. Simulation studies are conducted on affine and non-affine nonlinear systems, and further on the manipulator system, where all results have demonstrated the effectiveness of the proposed data-based approximate optimal control method.
\end{abstract}

Key words: Iterative neural dynamic programming (INDP); Data-based control; Approximate optimal control; Heuristic dynamic programming (HDP); Affine and non-affine nonlinear systems.

\section{Introduction}

* This paper was not presented at any IFAC meeting.

* Corresponding author H. He. Tel: +1-401-874-5844; Fax: +1-401-782-6422.

This work was supported in part by the National Natural Science Foundation of China under Grants 51529701, $61520106009,61533008, \mathrm{U} 1501251$ and 61533017 , in part by the US National Science Foundation under Grants ECCS 1053717 and IIS 1526835, in part by the National Aeronautics and Space Administration under Grant NNX15AK54A, in part by Beijing Natural Science Foundation under Grant 4162065. C. Mu and D. Wang make equal contributions to this work and share the first authorship. This work was done when $\mathrm{C}$. $\mathrm{Mu}$ was a Post-Doctoral Fellow at the Department of Electrical, Computer, and Biomedical Engineering, University of Rhode Island, Kingston, RI 02881, USA.

Email addresses: cxmu@tju.edu.edu (Chaoxu Mu), ding.wang@ia.ac.cn (Ding Wang), he@ele.uri.edu (Haibo He).

Preprint submitted to Automatica
Since nonlinear systems are widely existed in most industrial fields, the optimal control problem of nonlinear systems has attracted great attention in recent several decades. The nonlinear optimal control problem is usually formulated as coping with the nonlinear Hamilton-Jacobi-Bellman (HJB) equation, which is often difficult to be solved (Bellman, 1957; Lewis and Syrmos, 1995; Si, Barto, Powell and Wunsch, 2004). As is known to all, when the optimal control problem of linear systems is studied, the linear HJB equation can be evolved as the algebraic Riccati equation. The famous iterative solution strategy was proposed by converting the algebraic Riccati equation to a series of linear Lyapunov equations (Kleinman, 1968). Along this direction, the iterative solution strategy was extended to solve the approximate optimal control of a 
trainable manipulator in (Saridis and Lee, 1979). However, this iterative solution method only fits this kind of HJB equations that are linear partial differential equations. Motivated by these success, there has been a great deal of research developed to approximately solve the HJB equation with the great improvement of intelligent computation (Werbos, 1992; Beard, Saridis and Wen, 1997; Si et al., 2004; Wang, Liu, Wei, Zhao and Jin, 2012; Mu, Sun, Song and Yu, 2016). In (Werbos, 1992), an adaptive/approximate dynamic programming (ADP) algorithm was proposed to approximately solve optimal control problems in forward time by involving neural networks for function approximation. The generalized HJB equation was formulated to solve the optimal control problem from a view of successive approximation (Beard et al., 1997). For continuous-time nonlinear systems, a nearly constrained-optimal state feedback control method using a neural network HJB approach was given in (Abu-Khalaf and Lewis, 2004), and was extended to synchronous policy iteration in (Vamvoudakis and Lewis, 2010). Simultaneously, for discrete-time nonlinear systems, the iterative adaptive dynamic programming (IADP) strategy was improved to obtain the approximate solution of the nonlinear HJB equations by using neural networks (Al-Tamimi, Lewis and Abu-Khalaf, 2008; Zhang, Luo and Liu, 2009; Dierks, Thumati and Jagannathan, 2009; Wang, Jin, Liu and Wei, 2011; Wang, Mu and Liu, 2017). The value-iteration-based ADP algorithm was developed with several convergence results of both inner-loop and outer-loop iterations in (Heydari, 2014). In addition, there are several latest developments related to ADP, including approximation-error-based adaptive optimal control (Heydari, 2016), event-triggered optimal control design (Vamvoudakis, Mojoodi and Ferraz, 2016; Wang, Mu, Liu and Ma, 2016; Wang, Mu, He and Liu, 2016), ADP-based variable structure or switching control design (Heydari and Balakrishnan, 2014b; Fan and Yang, 2016; Mu, Ni, Sun and He, 2016a), cooperative control of multi-agent systems (Heydari and Balakrishnan, 2014a; Zhang, Liang, Wang and Feng, 2015), and so on.

In the industrial field, two prominent features are presented with the technological innovation and progress. One is that more and more real systems are facing the difficulty in establishing process models to support the control design due to increasing scales and complex operations. The other is that vast volume of data is stored during the industrial process but does not get used efficiently. Thus, the problem of data-based optimal control for nonlinear systems is significant and challenging. Recently, several data-based approximate optimal control approaches have been reported. For example, an online direct heuristic dynamic programming method was proposed by Si and Wang without requiring the controlled system model (Si and Wang, 2001), or was more specifically called neural dynamic programming (NDP), which was further developed to the tracking control problem of nonlinear systems (Yang, Si, Tsakalis and Rodriguez,
2009; Yang, Liu, Wang and Wei, 2014). The data-based online policy iteration approach was proposed to obtain adaptive optimal controllers for continuous-time linear systems with unknown system dynamics (Jiang and Jiang, 2012). A model-free approximate policy iteration method was developed based on a least-square weight updating for affine nonlinear continuous-time optimal control design (Luo, Wu, Huang and Liu, 2015). Based on the identification of neural networks, a datadriven robust approximate optimal control was designed for the tracking control of continuous-time general nonlinear systems (Zhang, Cui, Zhang and Luo, 2011). The robust ADP was studies for the robust optimal control design for a class of uncertain nonlinear systems (Jiang and Jiang, 2014).The approach of goal representation adaptive dynamic programming was proposed by adapting reinforcement signal ( $\mathrm{He}, \mathrm{Ni}$ and $\mathrm{Fu}, 2012$ ), which has been applied to tracking control problem (Mu, Ni, Sun and He, 2016b), maze navigation (Ni, He, Wen and $\mathrm{Xu}, 2013$ ) and power systems (Tang, Mu and He, 2016).

Compared with the NDP algorithm, this proposed method is an off-line algorithm by integrating the cost function iteration and the control law iteration into the NDP approach, while the NDP algorithm is with the merit of online learning and control. Compared with the iterative adaptive dynamic programming (IADP) method, the proposed method has built the data-based learning control framework by using a model network, while the IADP strategy is usually established on the known controlled system model and is particularly effective for affine nonlinear systems even a model network is utilized in this method (Wang et al., 2012; Wang, Liu, Zhang and Zhao, 2016). The contribution of this paper is summarized as follows. First, we propose the $\varepsilon$ optimal iterative ADP algorithm based on a prescribed error bound, where the convergence of the iterative algorithm as well as the equivalence of stopping criterion is proved from the view of theoretical analysis. Second, the INDP approach based on a HDP structure is developed to implement the data-based optimal control via estimating both the iterative control law and the iterative cost function. The novel design on the weight updating of the action neural network makes the implementation can be operated by only using system data, which has greatly improved the realization of the algorithm without involving the accurate system model. Third, by using a Lyapunov approach, the uniformly ultimately boundedness (UUB) stability is provided for the INDP controller.

This paper is organized as follows. In Section 2, the optimal control problem is formulated for general discretetime nonlinear systems. Section 3 presents the $\varepsilon$-optimal INDP algorithm and the iteration convergence analysis. The implementation strategy of INDP algorithm and the associated stability proof are provided in Section 4. In Section 5, three simulation examples are given to demonstrate the effectiveness of the proposed data-based INDP 
approximate optimal control scheme. Finally, we summarize this paper in Section 6 .

\section{Problem statement and preliminaries}

In this paper, the studied discrete-time nonlinear systems are generally described by

$$
x_{t+1}=F\left(x_{t}, u_{t}\right), t=0,1,2, \cdots
$$

where $x_{t}=\left[x_{1 t}, x_{2 t}, \cdots, x_{n t}\right]^{T} \in \mathbb{R}^{n}$ is the state vector at time step $t$, and $u_{t}=\left[u_{1 t}, u_{2 t}, \cdots, u_{m t}\right]^{T} \in \mathbb{R}^{m}$ is the control vector at time step $t$. The system function $F\left(x_{t}, u_{t}\right)$ is Lipschitz continuous on $\Omega_{x} \subseteq \mathbb{R}^{n}$ and $F(0,0)=0$.

Definition 1 (Werbos, 1992; Zhang et al., 2009) A nonlinear dynamical system is said to be stabilizable on a compact set $\Omega_{x} \subseteq \mathbb{R}^{n}$, if for any initial condition $x_{0} \in$ $\Omega_{x}$, there exists a control sequence $u_{0}, u_{1}, u_{2}, \cdots, u_{t} \in$ $\mathbb{R}^{m}$, such that the state $x_{t} \rightarrow 0$ as $t \rightarrow \infty$.

For the optimal control of discrete-time nonlinear system (1), it is expected to obtain an optimal control law $u_{t}$, which enables all the states of system (1) to stabilize at the origin and minimizes the following cost function $J\left(x_{t}\right)$,

$$
J\left(x_{t}\right)=\sum_{k=t}^{\infty} \beta^{k-t} R\left(x_{k}, u_{k}\right),
$$

where $R\left(x_{k}, u_{k}\right)$ is the utility function, $R\left(x_{k}, u_{k}\right) \geq 0$ for any $x_{k}$ and $u_{k}$, and $R(0,0)=0$. $\beta$ is the discount factor with $0<\beta \leq 1$. Generally speaking, the utility function can be chosen as the quadratic form of the states and the control variables, which is as follows:

$$
R\left(x_{k}, u_{k}\right)=x_{k}^{T} P x_{k}+u_{k}^{T} Q u_{k},
$$

where $P$ and $Q$ are symmetric positive definite matrices with appropriate dimensions.

A feedback control is used in this paper, such that $u_{t}=$ $u\left(x_{t}\right)$. The admissible control is introduced for the optimal control problem, which stabilizes system (1) at the origin and guarantees that the total cost function (2) is finite.

Definition 2 (Prokhorov, Santiago and Wunsch, 1995; Si et al., 2004) A feedback control $u_{t}$ defined on $\Omega_{x}$ is said to be admissible with respect to (2) if $u_{t}$ is continuous on a compact set $\Omega_{u} \subseteq \mathbb{R}^{m}, u(0)=0, u_{t}$ stabilizes system (1) on $\Omega_{x}$, and $J\left(x_{0}\right)$ is finite $\forall x_{0} \in \Omega_{x}$.

Note that the infinite-horizon cost function can be rewritten in a recursive form, then equation (2) is rewritten as

$$
\begin{aligned}
J\left(x_{t}\right) & =x_{t}^{T} P x_{t}+u_{t}^{T} Q u_{t}+\beta \sum_{k=t+1}^{\infty} \beta^{k-t-1} R\left(x_{k}, u_{k}\right) \\
& =x_{t}^{T} P x_{t}+u_{t}^{T} Q u_{t}+\beta J\left(x_{t+1}\right) .
\end{aligned}
$$

According to Bellman's optimality principle, the optimal cost function, denoted as $J^{*}\left(x_{t}\right)$, is time invariant and satisfies the discrete-time HJB equation, i.e.,

$$
J^{*}\left(x_{t}\right)=\min _{u_{t}}\left\{x_{t}^{T} P x_{t}+u_{t}^{T} Q u_{t}+\beta J^{*}\left(x_{t+1}\right)\right\} .
$$

In equation (5), it is observed that $J^{*}\left(x_{t}\right)$ is obtained by an optimal control law, denoted as $u_{t}^{*}$, which is able to minimize the total cost function $J\left(x_{t}\right)$ as $J^{*}\left(x_{t}\right)$. Based on this, the optimal control law $u_{t}^{*}$ is solved by the gradient of the right-hand side of equation (5) along $u_{t}$ as

$$
\frac{\partial\left(x_{t}^{T} P x_{t}+u_{t}^{T} Q u_{t}\right)}{\partial u_{t}}+\beta\left(\frac{\partial x_{t+1}}{\partial u_{t}}\right)^{T} \frac{\partial J^{*}\left(x_{t+1}\right)}{\partial x_{t+1}}=0 .
$$

Then, the optimal control feedback law $u_{t}^{*}$ is formulated as

$$
u_{t}^{*}=-\frac{\beta}{2} Q^{-1}\left(\frac{\partial x_{t+1}}{\partial u_{t}}\right)^{T} \frac{\partial J^{*}\left(x_{t+1}\right)}{\partial x_{t+1}} .
$$

We substitute equation (7) into equation (6), then the discrete-time HJB equation is obtained as

$$
\begin{aligned}
J^{*}\left(x_{t}\right)= & x_{t}^{T} P x_{t}+\frac{\beta^{2}}{4}\left(\frac{\partial J^{*}\left(x_{t+1}\right)}{\partial x_{t+1}}\right)^{T} \frac{\partial x_{t+1}}{\partial u_{t}} Q^{-1} \\
& \times\left(\frac{\partial x_{t+1}}{\partial u_{t}}\right)^{T} \frac{\partial J^{*}\left(x_{t+1}\right)}{\partial x_{t+1}}+\beta J^{*}\left(x_{t+1}\right),
\end{aligned}
$$

where $J^{*}\left(x_{t}\right)$ is the optimal cost function associated to the optimal control law $u_{t}^{*}$. From equation (7), it can be observed that the optimal cost function $J^{*}\left(x_{t+1}\right)$ is necessary for solving the optimal control $u_{t}^{*}$. However, it is impossible to obtain $x_{t+1}$ as well as the optimal cost function $J^{*}\left(x_{t+1}\right)$ at the current time step $t$. Therefore, on this occasion, it is admired that the approximate optimal solution of HJB equation is perused instead of the analytical optimal solution. In the following, we introduce the derivative of the INDP algorithm to approximate solve this kind of optimal control problems.

\section{$3 \varepsilon$-optimal INDP algorithm and iteration con- vergence analysis}

\subsection{Derivative of $\varepsilon$-optimal INDP algorithm}

The idea of iterative calculation for approximate solutions of HJB equation was presented in (Al-Tamimi et al., 2008; Zhang et al., 2009; Dierks et al., 2009; Wang et al., 2012; Wang, Liu, Zhang and Zhao, 2016). Based on 
Bellman's principle of optimality, the $\varepsilon$-optimal INDP algorithm is given as follows.

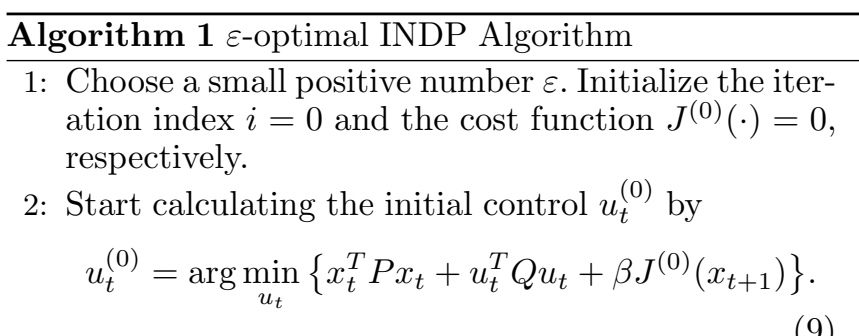

3: Once the control vector $u_{t}^{(0)}$ is obtained, update the iterative cost function as

$$
\begin{aligned}
J^{(1)}\left(x_{t}\right) & =\min _{u_{t}}\left\{x_{t}^{T} P x_{t}+u_{t}^{T} Q u_{t}+\beta J^{(0)}\left(F\left(x_{t}, u_{t}\right)\right)\right\} \\
& =x_{t}^{T} P x_{t}+\left(u_{t}^{(0)}\right)^{T} Q u_{t}^{(0)}
\end{aligned}
$$

4: Solve the control function by

$$
u_{t}^{(i)}=\arg \min _{u_{t}}\left\{R\left(x_{t}, u_{t}\right)+J^{(i)}\left(F\left(x_{t}, u_{t}\right)\right)\right\} .
$$

5: Update the cost function by

$$
\begin{aligned}
J^{(i+1)}\left(x_{t}\right) & =\min _{u_{t}}\left\{R\left(x_{t}, u_{t}\right)+J^{(i)}\left(F\left(x_{t}, u_{t}\right)\right)\right\} \\
& =R\left(x_{t}, u_{t}^{(i)}\right)+J^{(i)}\left(F\left(x_{t}, u_{t}^{(i)}\right)\right) .
\end{aligned}
$$

6: If $\left\|J^{(i+1)}\left(x_{t}\right)-J^{(i)}\left(x_{t}\right)\right\| \leq \varepsilon$, stop and obtain the approximate optimal control law $u_{t}^{(i)}$; else, set $i=$ $i+1$ and go to step 4 .

In the above iterative algorithm, we first assume that the initial cost value $J^{(0)}(\cdot)=0$ is known, and then start calculating the control law based on the known cost value. This algorithm is called as the value iteration, where $i$ is the iteration index and $t$ is the time index related to system states. The iterative cost function and the iterative control law are updated until $\left\|J^{(i+1)}\left(x_{t}\right)-J^{(i)}\left(x_{t}\right)\right\| \leq$ $\varepsilon$ is satisfied. $\|\cdot\|$ denotes the 2 -norm for a vector and the absolute value for a scalar through the paper. In the following, the convergence of the iterative algorithm between equations (11) and (12) will be addressed.

\subsection{Convergence analysis of E-optimal INDP algorithm}

Before starting the convergence analysis, we first review two lemmas.

Lemma 1 (Zhang et al., 2009; Wang et al., 2012) For the iterative cost function $J^{(i)}\left(x_{t}\right)$, as defined in equation (12), if system (1) is controllable, then there exists an upper bound $\Theta$ such that $0 \leq J^{(i)}\left(x_{t}\right) \leq \Theta$ for any $i$.
Lemma 2 (Wang and Liu, 2013) Assume that $\mu_{t}^{(i)}$ is an arbitrary control vector, and $u_{t}^{(i)}$ is the iterative control vector according to equation (11). The cost function associated with $\mu_{t}^{(i)}$ is defined as $\Xi^{(i)}\left(x_{t}\right)$ with the following expression

$$
\begin{aligned}
\Xi^{(i+1)}\left(x_{t}\right)= & x_{t}^{T} P x_{t}+\left(\mu_{t}^{(i)}\right)^{T} Q \mu_{t}^{(i)} \\
& +\beta \Xi^{(i)}\left(F\left(x_{t}, \mu_{t}^{(i)}\right)\right),
\end{aligned}
$$

and the iterative cost function associated with $u_{t}^{(i)}$ is denoted as $J^{(i)}\left(x_{t}\right)$, which can be formulated according to equation (12). If $J^{(0)}\left(x_{t}\right)=0$ and $\Xi^{(0)}\left(x_{t}\right)=0$, then $J^{(i+1)}\left(x_{t}\right) \leq \Xi^{(i+1)}\left(x_{t}\right)$ for any $i$.

Theorem 1 For the iterative cost function $J^{(i)}\left(x_{t}\right)$ with $J^{(0)}\left(x_{t}\right)=0$, defined in equation (12) and the iterative control law $u_{t}^{(i)}$ defined in equation (11), if the iterations are updated between equations (11) and (12), then as $i \rightarrow$ $\infty, J^{(i)}\left(x_{t}\right)$ converges to the optimal cost function defined in equation (5) and $u_{t}^{(i)}$ converges to the optimal control law defined in equation (7), i.e., $J^{(i)}\left(x_{t}\right) \rightarrow J^{*}\left(x_{t}\right)$ and $u_{t}^{(i)} \rightarrow u_{t}^{*}$ as $i \rightarrow \infty$.

PROOF. Based on Lemma 2, the cost function $\Xi^{(i)}\left(x_{t}\right)$ is defined in equation (13) with $\Xi^{(0)}\left(x_{t}\right)=0$, which is related to an arbitrary control vector $\mu_{t}^{(i-1)}$.

Now we show the relationship between $J^{(i)}\left(x_{t}\right)$ and $J^{(i+1)}\left(x_{t}\right)$ by mathematical induction. Take $\Xi^{(i)}\left(x_{t}\right)$ as the auxiliary variable. First, for $i=0$, as

$$
J^{(1)}\left(x_{t}\right)-\Xi^{(0)}\left(x_{t}\right)=x_{t}^{T} P x_{t}+\left(u_{t}^{(0)}\right)^{T} Q u_{t}^{(0)} \geq 0,
$$

it is obvious that $J^{(1)}\left(x_{t}\right) \geq \Xi^{(0)}\left(x_{t}\right)$. Second, for $i-1$, we assume that $J^{(i)}\left(x_{t}\right) \geq \Xi^{(i-1)}\left(x_{t}\right)$ holds for all $x_{t}$. Then, for $i$, it is able to obtain

$J^{(i+1)}\left(x_{t}\right)-\Xi^{(i)}\left(x_{t}\right)=\beta\left(J^{(i)}\left(x_{t+1}\right)-\Xi^{(i-1)}\left(x_{t+1}\right)\right) \geq 0$.

Therefore, in terms of mathematical induction, for all $i$, we can obtain $J^{(i+1)}\left(x_{t}\right) \geq \Xi^{(i)}\left(x_{t}\right)$. Combining with Lemma 2 that states $J^{(i)}\left(x_{t}\right) \leq \Xi^{(i)}\left(x_{t}\right)$, we have

$$
J^{(i+1)}\left(x_{t}\right) \geq \Xi^{(i)}\left(x_{t}\right) \geq J^{(i)}\left(x_{t}\right) .
$$

That is to say, $J^{(i)}\left(x_{t}\right)$ is nondecreasing satisfying $J^{(i)}\left(x_{t}\right) \leq J^{(i+1)}\left(x_{t}\right)$ for any $i$.

Considering Lemma 1, there exists an upper bound $\Theta$ for $0 \leq J^{(i)}\left(x_{t}\right) \leq \Theta$. It means that the limit of the iterative cost function exists, which is expressed as 
$\lim _{i \rightarrow \infty} J^{(i)}\left(x_{t}\right)=J^{(\infty)}\left(x_{t}\right)$. Then we will prove that $J^{(\infty)}\left(x_{t}\right)$ satisfies the following equation

$$
J^{(\infty)}\left(x_{t}\right)=\min _{u_{t}}\left\{x_{t}^{T} P x_{t}+u_{t}^{T} Q u_{t}+\beta J^{(\infty)}\left(x_{t+1}\right)\right\} .
$$

For any $u_{t}$ and $i$, according to (12), the cost function $J^{(i)}\left(x_{t}\right)$ can be written as

$$
J^{(i)}\left(x_{t}\right) \leq x_{t}^{T} P x_{t}+u_{t}^{T} Q u_{t}+\beta J^{(i-1)}\left(x_{t+1}\right) .
$$

Since $J^{(i)}\left(x_{t}\right) \leq J^{(\infty)}\left(x_{t}\right)$ for any $i$, based on equation (14), it can be deduced as

$$
J^{(i)}\left(x_{t}\right) \leq x_{t}^{T} P x_{t}+u_{t}^{T} Q u_{t}+\beta J^{(\infty)}\left(x_{t+1}\right), \forall i .
$$

Accompanying with $i \rightarrow \infty$, the following relationship can be derived from inequality (15)

$$
J^{(\infty)}\left(x_{t}\right) \leq x_{t}^{T} Q x_{t}+u_{t}^{T} R u_{t}+\beta J^{(\infty)}\left(x_{t+1}\right) .
$$

Due to $u_{t}$ is an arbitrary control vector, it implies that $J^{(\infty)}\left(x_{t}\right)$ satisfies

$$
J^{(\infty)}\left(x_{t}\right) \leq \min _{u_{t}}\left\{x_{t}^{T} Q x_{t}+u_{t}^{T} R u_{t}+\beta J^{(\infty)}\left(x_{t+1}\right)\right\} .
$$

Simultaneously, according to equation (12) in the iterative algorithm, $J^{(i)}\left(x_{t}\right)$ is formulated as

$$
J^{(i)}\left(x_{t}\right)=\min _{u_{t}}\left\{x_{t}^{T} Q x_{t}+u_{t}^{T} R u_{t}+\beta J^{(i-1)}\left(x_{t+1}\right)\right\}, \forall i .
$$

Since $J^{(i)}\left(x_{t}\right) \leq J^{(\infty)}\left(x_{t}\right)$, then we have the following inequality

$J^{(\infty)}\left(x_{t}\right) \geq \min _{u_{t}}\left\{x_{t}^{T} Q x_{t}+u_{t}^{T} R u_{t}+\beta J^{(i-1)}\left(x_{t+1}\right)\right\}, \forall i$.

Similarly, by letting $i \rightarrow \infty$, we can get

$$
J^{(\infty)}\left(x_{t}\right) \geq \min _{u_{t}}\left\{x_{t}^{T} Q x_{t}+u_{t}^{T} R u_{t}+\beta J^{(\infty)}\left(x_{t+1}\right)\right\} .
$$

Considering (16) and (17), it can be concluded that $J^{(\infty)}\left(x_{t}\right)$ satisfies

$$
J^{(\infty)}\left(x_{t}\right)=\min _{u_{t}}\left\{x_{t}^{T} Q x_{t}+u_{t}^{T} R u_{t}+\beta J^{(\infty)}\left(x_{t+1}\right)\right\} .
$$

Obviously, $J^{(\infty)}\left(x_{t}\right)$ is the solution of the discrete-time HJB equation. Considering the unique solution of the discrete-time HJB equation, it means that $J^{(\infty)}\left(x_{t}\right)$ in equation (18) and $J^{*}\left(x_{t}\right)$ in equation (5) are really the same. That is to say, $\lim _{i \rightarrow \infty} J^{(i)}\left(x_{t}\right)=J^{*}\left(x_{t}\right)$. Accordingly, according to (7) and (18), we can conclude that the corresponding control law also converges to the optimal one as $i \rightarrow \infty$. Thus, the proof is completed.
Remark 1 Though Theorem 1 provides the convergence analysis of the iteration algorithm, it is impossible to conduct $i \rightarrow \infty$. Therefore, the $\varepsilon$-optimal criterion is given in the following theorem, which states that the $\varepsilon$-optimal criterion is equivalent to the infinite-horizon optimal criterion.

Theorem 2 If system (1) is controllable and starts from an arbitrary state $x_{t}$, the iterative cost function $J^{(i)}\left(x_{t}\right)$ is defined by (12) and $J^{*}\left(x_{t}\right)$ is the optimal cost function, then after a finite iteration number $i$, the following $\varepsilon$ optimal iteration criterion

$$
\left\|J^{(i+1)}\left(x_{t}\right)-J^{(i)}\left(x_{t}\right)\right\| \leq \varepsilon
$$

is equal to the infinite-horizon optimal iteration criterion

$$
\left\|J^{(i)}\left(x_{t}\right)-J^{*}\left(x_{t}\right)\right\| \leq \varepsilon,
$$

where $\varepsilon>0$ is a preset iterative tolerable error.

PROOF. This proof wants to show the above two conditions are equivalent. In terms of the analysis that $J^{(i)}\left(x_{t}\right)$ is nondecreasing in Theorem 1, the basic inequalities are listed as follows:

$$
J^{(*)}\left(x_{t}\right) \geq J^{(i+1)}\left(x_{t}\right) \geq J^{(i)}\left(x_{t}\right)
$$

On one hand, if $\left\|J^{(*)}\left(x_{t}\right)-J^{(i)}\left(x_{t}\right)\right\| \leq \varepsilon$ is established, according to inequalities (21), then we can obtain

$$
J^{(*)}\left(x_{t}\right)-J^{(i)}\left(x_{t}\right) \leq \varepsilon .
$$

By introducing $J^{(i+1)}\left(x_{t}\right)$ into the above inequality, it has

$$
J^{(i)}\left(x_{t}\right) \leq J^{(i+1)}\left(x_{t}\right) \leq J^{(*)}\left(x_{t}\right) \leq J^{(i)}\left(x_{t}\right)+\varepsilon .
$$

That means that $J^{(i+1)}\left(x_{t}\right)$ satisfies the relation

$$
J^{(i)}\left(x_{t}\right) \leq J^{(i+1)}\left(x_{t}\right) \leq J^{(i)}\left(x_{t}\right)+\varepsilon \text {. }
$$

Therefore, we have

$$
\left\|J^{(i+1)}\left(x_{t}\right)-J^{(i)}\left(x_{t}\right)\right\| \leq \varepsilon .
$$

On the other hand, if the condition $\| J^{(i+1)}\left(x_{t}\right)-$ $J^{(i)}\left(x_{t}\right) \| \leq \varepsilon$ is established, since $J^{(i)}\left(x_{t}\right)$ is nondecreasing, then we have

$$
J^{(i)}\left(x_{t}\right) \geq J^{(i+1)}\left(x_{t}\right)-\varepsilon .
$$

Based on inequalities (21) and (23), the relationship between $J^{*}\left(x_{t}\right)$ and $J^{(i+1)}\left(x_{t}\right)$ can be indicated as

$$
J^{*}\left(x_{t}\right) \geq J^{(i+1)}\left(x_{t}\right)-\varepsilon .
$$


Simultaneously, it is obvious that $J^{(i+1)}\left(x_{t}\right)-J^{*}\left(x_{t}\right) \leq$ $\varepsilon$. Therefore, we have the following derivation from the above inequality

$$
\left\|J^{*}\left(x_{t}\right)-J^{(i+1)}\left(x_{t}\right)\right\| \leq \varepsilon
$$

Based on the analysis from the two sides, it can be concluded that

$\left\|J^{(i+1)}\left(x_{t}\right)-J^{(i)}\left(x_{t}\right)\right\| \leq \varepsilon \Longleftrightarrow\left\|J^{(i)}\left(x_{t}\right)-J^{*}\left(x_{t}\right)\right\| \leq \varepsilon$,

which thus implements the proof.

Considering that $J^{*}\left(x_{t}\right)$ is not really known in the implementation, the $\varepsilon$-optimal iteration criterion provides an operable condition which is related to the preset tolerant error. Then, we will clearly present the implementation of the INDP algorithm.

\section{$4 \varepsilon$-optimal INDP algorithm based on the HDP structure and system data}

In the above iterative algorithm, $u_{t}^{(i)}$ and $J^{(i)}\left(x_{t}\right)$ is usually obtained by using the function approximation approach. This algorithm is implemented by neural networks based on the HDP structure which can be operated without the accurate system model. Therefore, it is a data-driven approximate optimal control algorithm. Fig. 1 shows the implementation architecture for the INDP algorithm. It contains four neural networks: one model network, one action network, and two critic networks. Note that the two critic networks presented in the figure show the time difference during the algorithmic procedure, and they actually are the same network.

\subsection{The model neural network}

The dynamics of the controlled system are assumed to be unknown. A three-layer neural network is used to identify the system dynamics based on the input-output data. Before starting the $\varepsilon$-optimal iterative algorithm, the model neural network is first trained.

Considering the general nonlinear system in equation (1), at the time step $t$, the current system states $x_{t}$ and control inputs $u_{t}$ are used as the inputs of the model network, then the estimation of $x_{t+1}$ is outputted. With $h_{m}$ hidden layer neurons, the input-to-hidden weights $s_{m} \in \mathbb{R}^{(n+m) \times h_{m}}$ are randomly initialized and then are kept unchanged, while the optimal hidden-to-output weight vector is denoted as $\nu \in \mathbb{R}^{h_{m} \times n}$. A bounded activation function $\vartheta_{m}(\kappa) \in \mathbb{R}^{h_{m}}$ is used, where $\kappa$ expresses a general independent-variable of the activation function. Based on the universal approximation theory of neural networks, the system state vector of the next time step $x_{t+1}$ can be formulated by

$$
x_{t+1}=\nu^{T} \vartheta_{m}\left(s_{m}^{T} \theta_{t}\right)+\zeta_{m t},
$$

where $\theta_{t}=\left[x_{t}^{T}, u_{t}^{T}\right]^{T}$ is the input vector, and $\zeta_{m t}$ is the reconstruction error of the model neural network. Let $\sigma_{t}=s_{m}^{T} \theta_{t} \in \mathbb{R}^{h_{m}}$, therefore equation (25) can be simplified as $x_{t+1}=\nu^{T} \vartheta_{m}\left(\sigma_{t}\right)+\zeta_{m t}$.

Since the optimal weight vector $\nu$ is not really known, we use the approximate weight vector $\hat{\nu}_{t}$ to approximate the optimal weight vector $\nu$, such that the next system state vector can be estimated as

$$
\hat{x}_{t+1}=\hat{\nu}_{t}^{T} \vartheta_{m}\left(\sigma_{t}\right)
$$

where $\hat{\nu}_{t}$ is updated by minimizing the identification error to approach $\nu$ as much as possible.

The identification error of the model network is defined as $\tilde{x}_{t+1}=\hat{x}_{t+1}-x_{t+1}$. According to equations (25) and (26), $\tilde{x}_{t+1}$ can be deduced as

$$
\begin{aligned}
\tilde{x}_{t+1} & =\hat{\nu}_{t}^{T} \vartheta_{m}\left(\sigma_{t}\right)-\nu^{T} \vartheta_{m}\left(\sigma_{t}\right)-\zeta_{m t} \\
& =\tilde{\nu}_{t}^{T} \vartheta_{m}\left(\sigma_{t}\right)-\zeta_{m t}=\xi_{m t}-\zeta_{m t}
\end{aligned}
$$

where $\tilde{\nu}_{t}=\hat{\nu}_{t}-\nu, \xi_{m t}=\tilde{\nu}_{t}^{T} \vartheta_{m}\left(\sigma_{t}\right)$. Based on the definition of identification error, the model network error is denoted as

$$
E_{m t}=\frac{1}{2} \tilde{x}_{t+1}^{T} \tilde{x}_{t+1}
$$

The gradient-based descent approach is used for weight updating during the back-propagation process, which is

$$
\begin{aligned}
\hat{\nu}_{t+1} & =\hat{\nu}_{t}-\eta_{m}\left(\frac{\partial E_{m t}}{\partial \hat{\nu}_{t}}\right) \\
& =\hat{\nu}_{t}-\eta_{m} \vartheta_{m}\left(\sigma_{t}\right) \tilde{x}_{t+1}^{T}
\end{aligned}
$$

where $\eta_{m}>0$ is the learning rate of the model neural network.

Lemma 3 In terms of the property of matrix trace, the useful equalities are given as follows:

(1) A matrix $X$ and its transpose have the same trace, i.e. $\operatorname{tr}\{X\}=\operatorname{tr}\left\{X^{T}\right\}$

(2) The matrices in a trace of a product can be switched: if $A$ is an $m_{1} \times n_{1}$ matrix, $B$ is an $n_{1} \times m_{2} m a-$ trix, $C$ is an $m_{2} \times n_{1}$ matrix and $D$ is an $n_{1} \times$ $m_{1}$ matrix, then the trace is invariant under cyclic permutations, i.e., $\operatorname{tr}\{A B C D\}=\operatorname{tr}\{B C D A\}=$ $\operatorname{tr}\{C D A B\}=\operatorname{tr}\{D A B C\}$. 
Fig. 1. The implementation architecture of the INDP algorithm with the HDP structure.

Lemma 4 (Dierks et al., 2009) For vectors a and b, the Cauchy-Schwarz inequality can be applied as

$$
(a+b)^{T}(a+b) \leq 2\left(a^{T} a+b^{T} b\right) .
$$

Assumption 1 Both the activation function $\vartheta_{m}(\cdot)$ and the reconstruction error $\zeta_{m t}$ are bounded, i.e., $\left\|\vartheta_{m}(\cdot)\right\| \leq$ $\vartheta_{M}$ and $\left\|\zeta_{m t}\right\| \leq \zeta_{M}$, where $\vartheta_{M}$ and $\zeta_{M}$ are positive constants.

The following theorem clarifies the stability of the identification error $\tilde{x}_{t+1}$ for the model network.

Theorem 3 For the model network described by equation (26), if Assumption 1 is satisfied and the weight vector $\hat{\nu}_{t}$ is updated as equation (29), then the identification error $\tilde{x}_{t}$ are uniformly ultimately bounded.

PROOF. Choose a positive Lyapunov function as

$$
L_{1}(t)=\tilde{x}_{t}^{T} \tilde{x}_{t}+\eta_{m}^{-1} \operatorname{tr}\left\{\tilde{\nu}_{t}^{T} \tilde{\nu}_{t}\right\} .
$$

The first difference of $L_{1}(t)$ is denoted as $\Delta L_{1}(t)=$ $L_{1}(t+1)-L_{1}(t)$, then $\Delta L_{1}(t)$ is

$$
\Delta L_{1}(t)=\tilde{x}_{t+1}^{T} \tilde{x}_{t+1}-\tilde{x}_{t}^{T} \tilde{x}_{t}+\eta_{m}^{-1} \operatorname{tr}\left\{\tilde{\nu}_{t+1}^{T} \tilde{\nu}_{t+1}-\tilde{\nu}_{t}^{T} \tilde{\nu}_{t}\right\} .
$$

Equation (29) provides the relation between $\tilde{\nu}_{t+1}$ and $\tilde{\nu}_{t}$, which can be substituted into equation (31) to obtain

$$
\begin{aligned}
\Delta L_{1}(t)= & \tilde{x}_{t+1}^{T} \tilde{x}_{t+1}-\tilde{x}_{t}^{T} \tilde{x}_{t}+\operatorname{tr}\left\{\eta_{m} \tilde{x}_{t+1} \vartheta_{m}^{T}\left(\sigma_{t}\right) \times\right. \\
& \vartheta_{m}\left(\sigma_{t}\right) \tilde{x}_{t+1}^{T}-\tilde{\nu}_{t+1}^{T} \vartheta_{m}\left(\sigma_{t}\right) \tilde{x}_{t+1}^{T} \\
& \left.-\tilde{x}_{t+1} \vartheta_{m}^{T}\left(\sigma_{t}\right) \tilde{\nu}_{t+1}\right\} .
\end{aligned}
$$

By applying the trace operation in Lemma 3, we have $\operatorname{tr}\left\{\tilde{\nu}_{t+1}^{T} \vartheta_{m}\left(\sigma_{t}\right) \tilde{x}_{t+1}^{T}\right\}=\operatorname{tr}\left\{\tilde{x}_{t+1} \vartheta_{m}^{T}\left(\sigma_{t}\right) \tilde{\nu}_{t+1}\right\}, \operatorname{tr}\left\{\tilde{x}_{t+1}\right.$ $\left.\vartheta_{m}^{T}\left(\sigma_{t}\right) \vartheta_{m}\left(\sigma_{t}\right) \tilde{x}_{t+1}^{T}\right\}=\operatorname{tr}\left\{\vartheta_{m}^{T}\left(\sigma_{t}\right) \vartheta_{m}\left(\sigma_{t}\right) \tilde{x}_{t+1} \tilde{x}_{t+1}^{T}\right\}$, then $\Delta L_{1}(t)$ can be derived as

$$
\begin{aligned}
\Delta L_{1}(t)= & \tilde{x}_{t+1}^{T} \tilde{x}_{t+1}-\tilde{x}_{t}^{T} \tilde{x}_{t}+\eta_{m} \vartheta_{m}^{T}\left(\sigma_{t}\right) \vartheta_{m}\left(\sigma_{t}\right) \times \\
& \operatorname{tr}\left\{\tilde{x}_{t+1} \tilde{x}_{t+1}^{T}\right\}-2 \operatorname{tr}\left\{\tilde{x}_{t+1} \vartheta_{m}^{T}\left(\varphi_{t}\right) \tilde{\nu}_{t+1}\right\}
\end{aligned}
$$

By introducing $\tilde{x}_{t+1}=\xi_{m t}-\zeta_{m t}$, applying the CauchySchwarz inequality in Lemma 4 to the term $\tilde{x}_{t+1}^{T} \tilde{x}_{t+1}$, and using Assumption 1, $\Delta L_{1}(t)$ can be further deduced as

$$
\begin{aligned}
\Delta L_{1}(t) \leq & \tilde{x}_{t+1}^{T} \tilde{x}_{t+1}-\tilde{x}_{t}^{T} \tilde{x}_{t}-2 \operatorname{tr}\left\{\left(\xi_{m t}-\zeta_{m t}\right) \xi_{m t}^{T}\right\} \\
& +2 \eta_{m} \vartheta^{T}\left(\varphi_{t}\right) \vartheta\left(\varphi_{t}\right) \operatorname{tr}\left\{\xi_{m t}^{T} \xi_{m t}+\zeta_{m t}^{T} \zeta_{m t}\right\} \\
= & \xi_{m t}^{T} \xi_{m t}-2 \xi_{m t}^{T} \zeta_{m t}+\zeta_{m t}^{T} \zeta_{m t}-\tilde{x}_{t}^{T} \tilde{x}_{t} \\
& -2 \xi_{m t}^{T} \xi_{m t}+2 \xi_{m t}^{T} \zeta_{m t}+2 \eta_{m} \vartheta_{m}^{T}\left(\varphi_{t}\right) \vartheta_{m}\left(\varphi_{t}\right) \times \\
& \left(\xi_{m t}^{T} \xi_{m t}+\zeta_{m t}^{T} \zeta_{m t}\right) \\
\leq & -\xi_{m t}^{T} \xi_{m t}+\zeta_{m t}^{T} \zeta_{m t}-\tilde{x}_{t}^{T} \tilde{x}_{t}+2 \eta_{m} \vartheta_{M}^{2} \xi_{m t}^{T} \xi_{m t} \\
& +2 \eta_{m} \vartheta_{M}^{2} \zeta_{m t}^{T} \zeta_{m t} \\
\leq & -\left(1-2 \eta_{m} \vartheta_{M}^{2}\right) \xi_{m t}^{T} \xi_{m t}+\left(1+2 \eta_{m} \vartheta_{M}^{2}\right) \zeta_{M}^{2} \\
& -\tilde{x}_{t}^{T} \tilde{x}_{t} .
\end{aligned}
$$

From inequality (34), if $\eta_{m}$ satisfies $0<\eta_{m}<1 / 2 \vartheta_{M}^{2}$, then we have

$$
\Delta L_{1}(t) \leq\left(1+2 \eta_{m} \vartheta_{M}^{2}\right) \zeta_{M}^{2}-\tilde{x}_{t}^{T} \tilde{x}_{t}
$$

Therefore, $\Delta L_{1}(t) \leq 0$ if

$$
\left\|\tilde{x}_{t}\right\| \geq \sqrt{1+2 \eta_{m} \vartheta_{M}^{2}} \zeta_{M}
$$

with $0<\eta_{m}<1 / 2 \vartheta_{M}^{2}$. Based on the Lyapunov stability theorem, the identification error $\tilde{x}_{t}$ is uniformly ultimately bounded. Thus the theorem is proved.

\subsection{The critic neural network}

The critic network is used to approximate the iterative cost function. The three-layer neural network is employed to implement the critic network, which includes $n$ input neurons, $h_{c}$ hidden layer neurons and one output neuron. The activation function $\vartheta_{c}(\kappa) \in \mathbb{R}^{h_{c}}$ is used in the critic network. In the $i$-th iteration, two critic networks have the same input-to-hidden and hiddento-output weight vectors, denoted as $s_{c} \in \mathbb{R}^{n \times h_{c}}$ and 
$\hat{v}_{t} \in \mathbb{R}^{h_{c}}$, where $s_{c}$ is unchanged and only $\hat{v}_{t}$ is updated. For the first critic network, it takes $x_{t}$ as the input vector and outputs $\hat{J}^{(i+1)}\left(x_{t}\right)$ as

$$
\hat{J}^{(i+1)}\left(x_{t}\right)=\hat{v}_{t}^{T} \vartheta_{c}\left(s_{c}^{T} x_{t}\right)=\hat{v}_{t}^{T} \vartheta_{c}\left(z_{t}\right),
$$

where $z_{t}=s_{c}^{T} x_{t} \cdot \hat{J}^{(i+1)}\left(x_{t}\right)$ is the estimated iterative cost function to approach $J^{(i+1)}\left(x_{t}\right)$.

The model network outputs $\hat{x}_{t+1}$, which is used as the input vector of the second critic network. The hiddento-output weight vector $\hat{v}_{t}$ is transmitted from the first critic network and then is used to calculate $\hat{J}^{(i)}\left(\hat{x}_{t+1}\right)$ as

$$
\hat{J}^{(i)}\left(\hat{x}_{t+1}\right)=\hat{v}_{t}^{T} \vartheta_{c}\left(s_{c}^{T} \hat{x}_{t+1}\right)
$$

With the estimated $\hat{J}^{(i)}\left(\hat{x}_{t+1}\right)$ and $\hat{u}_{t}$, the target iterative cost function be calculated by

$$
J^{(i+1)}\left(x_{t}\right)=x_{t}^{T} P x_{t}+\hat{u}_{t}^{T} Q \hat{u}_{t}+\beta \hat{J}^{(i)}\left(\hat{x}_{t+1}\right),
$$

then the error function of the critic network is defined as $E_{c t}$, and the critic network is trained by the following objective function

$$
\begin{aligned}
& \min _{\hat{v}_{t}} E_{c t}=\min _{\hat{v}_{t}} \frac{1}{2} e_{c t}^{T} e_{c t}, \\
& e_{c t}=\hat{J}^{(i+1)}\left(x_{t}\right)-J^{(i+1)}\left(x_{t}\right) .
\end{aligned}
$$

Based on equation (39), using the gradient-based adaptation algorithm, the weight updating rule for the critic network is given as follows:

$$
\begin{aligned}
\hat{v}_{t+1} & =\hat{v}_{t}-\eta_{c} \frac{\partial E_{c t}}{\partial v_{t}} \\
\frac{\partial E_{c t}}{\partial v_{t}} & =\frac{\partial E_{c t}}{\partial e_{c t}} \frac{\partial e_{c t}}{\partial \hat{J}^{(i+1)}\left(x_{t}\right)} \frac{\partial \hat{J}^{(i+1)}\left(x_{t}\right)}{\partial \hat{v}_{t}} \\
& =e_{c t} \vartheta_{c}\left(s_{c}^{T} x_{t}\right),
\end{aligned}
$$

where $\eta_{c}>0$ is the learning rate of the critic network. The above weight updating rule is only applied for the first critic network, the weight vector of the second critic network is transmitted from the first one without the weight updating calculation.

\subsection{The action neural network}

In the HDP implementation, the action network with $h_{a}$ hidden layer neurons is constructed to approach the iterative control law. In the $i$-th iteration, the inputto-hidden weight vector is randomly initialized as $s_{a} \in$ $\mathbb{R}^{n \times h_{a}}$ and is kept unchanged, and the hidden-to-output weight vector is denoted as $\omega_{t} \in \mathbb{R}^{h_{a} \times m}$. The action network takes $x_{t}$ as the inputs, and outputs $\hat{u}_{t}$ to approximate $u_{t}^{(i)}$. With the bounded activation function $\vartheta_{a}(\kappa) \in \mathbb{R}^{h_{a}}, \hat{u}_{t}$ is expressed as

$$
\hat{u}_{t}=\hat{\omega}_{t}^{T} \vartheta_{a}\left(s_{a}^{T} x_{t}\right)=\hat{\omega}_{t}^{T} \vartheta_{a}\left(\varsigma_{t}\right)
$$

where $\varsigma_{t}=s_{a}^{T} x_{t} \in \mathbb{R}^{h_{a}}$.

The action network is trained through minimizing the network error between the ultimate objective $U_{C}$ and the estimated iterative cost function $\hat{J}^{(i)}\left(\hat{x}_{t+1}\right)$. The weight vector $\hat{\omega}_{t}$ is regulated by the error back propagation method. The objective function of the action network is defined as

$$
\begin{gathered}
\min _{\hat{\omega}_{t}} E_{a t}=\min _{\hat{\omega}_{t}} \frac{1}{2} e_{a t}^{T} e_{a t}, \\
e_{a t}=\hat{J}^{(i)}\left(\hat{x}_{t+1}\right)-U_{C},
\end{gathered}
$$

where $U_{C}=0$ is the ultimate cost objective for all $i$ and $t$. Similarly, according to the gradient-based adaptation strategy and the chain derivation rule, the weight vector of the action network is updated as

$$
\begin{aligned}
& \hat{\omega}_{(t+1)}=\hat{\omega}_{t}-\eta_{a} \frac{\partial E_{a t}}{\partial \hat{\omega}_{t}}, \\
& \frac{\partial E_{a t}}{\partial \hat{\omega}_{t}}=\frac{\partial E_{a t}}{\partial e_{a t}} \frac{\partial e_{a t}}{\partial \hat{J}^{(i)}\left(\hat{x}_{t+1}\right)} \frac{\partial \hat{J}^{(i)}\left(\hat{x}_{t+1}\right)}{\partial \hat{x}_{t+1}} \frac{\hat{x}_{t+1}}{\partial \hat{u}_{t}} \frac{\partial \hat{u}_{t}}{\hat{\omega}_{t}},
\end{aligned}
$$

where $\eta_{a}>0$ is the learning rate of the action network. If the tangent function is used as the activation function for all the three networks, i.e., $\vartheta_{m}\left(\sigma_{t}\right), \vartheta_{c}\left(z_{t}\right)$ and $\vartheta_{a}\left(\varsigma_{t}\right)$ are all tansig functions. $\kappa_{j}$ means one component of the general independent-variable $\kappa$. Accordingly, it can be replaced as $\sigma_{t, j}$ for the model network, $z_{t, j}$ for the critic network and $\varsigma_{t, j}$ for the action network. In order to uniformly describe all the activation functions of three networks, a general subscript $\ell$ is introduced, such that any component of the activation functions can be uniformly expressed as

$$
\vartheta_{\ell}\left(\kappa_{j}\right)=\frac{1-e^{-\kappa_{j}}}{1+e^{-\kappa_{j}}}
$$

where $\ell$ is replaceable by $m, c$ and $a$ for model, critic and action networks, respectively. The derivative of $\vartheta_{\ell}\left(\kappa_{j}\right)$ is

$$
\frac{\mathrm{d} \vartheta_{\ell}\left(\kappa_{j}\right)}{\mathrm{d} \kappa_{j}}=\frac{1}{2}\left(1-\vartheta_{\ell}^{2}\left(\kappa_{j}\right)\right),
$$

then $\partial \hat{J}^{(i)}\left(\hat{x}_{t+1}\right) / \partial \hat{x}_{t+1}$ and $\partial \hat{x}_{t+1} / \partial \hat{u}_{t}^{(i)}$ can be separately obtained as

$$
\begin{aligned}
& \frac{\partial \hat{J}^{(i)}\left(\hat{x}_{t+1}\right)}{\partial \hat{x}_{t+1}}=\hat{v}_{t}^{T} \frac{\partial \vartheta_{c}\left(z_{t+1}\right)}{\partial z_{t+1}} \frac{\partial z_{t+1}}{\partial \hat{x}_{t+1}}=\hat{v}_{t}^{T} \varphi_{t+1} s_{c}^{T}, \\
& \frac{\partial \hat{x}_{t+1}}{\partial \hat{u}_{t}}=\hat{\nu}_{t}^{T} \frac{\partial \vartheta_{m}\left(\sigma_{t}\right)}{\partial \sigma_{t}} \frac{\partial \sigma_{t}}{\partial \theta_{t}} \frac{\partial \theta_{t}}{\partial \hat{u}_{t}}=\hat{\nu}_{t}^{T} \psi_{t} s_{m}^{T} \rho,
\end{aligned}
$$


where $z_{t+1}=s_{c}^{T} x_{t+1} \cdot \varphi_{t+1} \in \mathbb{R}^{h_{c} \times h_{c}}, \psi_{t} \in \mathbb{R}^{h_{m} \times h_{m}}$ and $\rho \in \mathbb{R}^{(n+m) \times m}$ are expressed as

$$
\begin{gathered}
\varphi_{t+1}=\frac{1}{2}\left[\begin{array}{ccc}
1-\vartheta_{c}^{2}\left(z_{t+1,1}\right) & \cdots & 1-\vartheta_{c}^{2}\left(z_{t+1, h_{c}}\right) \\
\vdots & \ddots & \vdots \\
1-\vartheta_{c}^{2}\left(z_{t+1,1}\right) & \cdots & 1-\vartheta_{c}^{2}\left(z_{t+1, h_{c}}\right)
\end{array}\right], \\
\psi_{t}=\frac{1}{2}\left[\begin{array}{ccc}
1-\vartheta_{m}^{2}\left(\sigma_{t, 1}\right) & \cdots & 1-\vartheta_{m}^{2}\left(\sigma_{t, h_{m}}\right) \\
\vdots & \ddots & \vdots \\
1-\vartheta_{m}^{2}\left(\sigma_{t, 1}\right) & \cdots & 1-\vartheta_{m}^{2}\left(\sigma_{t, h_{m}}\right)
\end{array}\right], \rho=\left[\begin{array}{l}
0_{n \times m} \\
I_{m \times m}
\end{array}\right] .
\end{gathered}
$$

Therefore, $\partial E_{a t} / \partial \hat{\omega}_{t}$ can be specifically formulated as

$$
\begin{aligned}
\frac{\partial E_{a t}}{\partial \hat{\omega}_{t}} & =\vartheta_{a}\left(\varsigma_{t}\right) e_{a t} \hat{v}_{t}^{T} \varphi_{t} s_{c}^{T} \hat{\nu}_{t}^{T} \psi_{t} s_{m}^{T} \rho \\
& =\vartheta_{a}\left(\varsigma_{t}\right) \hat{v}_{t}^{T} \vartheta_{c}\left(z_{t+1}\right) \hat{v}_{t}^{T} \varphi_{t} s_{c}^{T} \hat{\nu}_{t}^{T} \psi_{t} s_{m}^{T} \rho \\
& =\vartheta_{a}\left(\varsigma_{t}\right) \hat{v}_{t}^{T} \vartheta_{c}\left(z_{t+1}\right) \hat{v}_{t}^{T} \varphi_{t} \pi
\end{aligned}
$$

where $\pi=s_{c}^{T} \hat{\nu}_{t}^{T} \psi_{t} s_{m}^{T} \rho, \pi \in \mathbb{R}^{h_{c} \times m}$ and is kept as a constant matrix after the model network is well trained.

\subsection{Algorithm procedure}

The $\varepsilon$-optimal INDP algorithm based on the HDP structure is summarized as follows.

\subsection{Stability analysis}

It has been proved that if the input-to-hidden weight vector is randomly initially chosen and is unchanged, then the neural network estimation error can be made arbitrarily small with the sufficiently large number of hidden layer neurons and bound activation functions (Igelnik and Pao, 1995). Therefore, in the proposed INDP algorithm, only the hidden-output weight vectors in all neural networks are designed. The stability of the proposed algorithm is now investigated.

For simple representation, let $\vartheta_{a t} \in \mathbb{R}^{h_{a}}$ and $\vartheta_{c t} \in$ $\mathbb{R}^{h_{c}}$ denote activation functions for the action network and the critic network without arguments, respectively, $\left\|\vartheta_{a t}\right\|^{2}=\vartheta_{a t}^{T} \vartheta_{a t}$ and $\left\|\vartheta_{c t}\right\|^{2}=\vartheta_{c t}^{T} \vartheta_{c t}$. The estimated weights are denoted as $\hat{\omega}_{t}$ and $\hat{v}_{t}$ for the action network and the critic network, respectively, while the associated optimal weight vectors are denoted as $\omega$ and $v$. Correspondingly, the weight errors are defined as $\tilde{\omega}_{t}=\hat{\omega}_{t}-\omega$ and $\tilde{v}_{t}=\hat{v}_{t}-v$. In the INDP algorithm, $J^{(i+1)}\left(x_{t}\right)$ in equation (38) is regard as the target iterative cost function, therefore it can be expressed by

$$
J^{(i+1)}\left(x_{t}\right)=v^{T} \vartheta_{c t}+\zeta_{c t},
$$

where $\vartheta_{c t}=\vartheta_{c}\left(z_{t}\right), \zeta_{c t}$ denotes the arbitrarily small reconstruction error. In the following, the Lyapunov stability analysis is used to prove the UUB stability of weight $\overline{\text { Algorithm } 2 \text { Implementation Outline for } \varepsilon \text {-optimal }}$ INDP Algorithm Based on the HDP Structure

1: Set the parameters $\varepsilon, \varepsilon_{m}, \varepsilon_{a}, \varepsilon_{c}, \eta_{m}, \eta_{a}, \eta_{c}, N_{i}, N_{m}$, $N_{a}, N_{c}, \beta, P$ and $Q$.

2: Randomly initialize weight vector matrices $s_{m}, s_{c}$, $s_{a}, \hat{\nu}_{0}, \hat{v}_{0}$ and $\hat{\omega}_{0}$ in given intervals.

3: Train the model network

(1) Construct a three-layer neural network as the model network with the initial weight vectors $s_{m}$ and $\hat{\nu}_{0}$.

(2) Train the model network with measured system data for maximal $N_{m}$ steps or until $E_{m t} \leq \varepsilon_{m}$ is satisfied.

(3) Obtain the trained weight vector $\hat{\nu}_{t}$ and $s_{m}$, and then maintain them during the algorithm.

4: Set the iterative index $i=0$ and the initial iterative cost function $J^{(0)}(\cdot)=0$. According to equation (9), calculate the initial control $u_{t}^{(0)}$.

5: Use $s_{c}$ and $\hat{v}_{0}$ as the input-to-hidden and hidden-tooutput weight vectors of the critic network, compute $\hat{J}^{(1)}\left(x_{t}\right)$ and $J^{(1)}\left(x_{t}\right)$ by equations (36) and (38), respectively. Train the critic network for maximal $N_{c}$ steps with equation (40) or until $E_{c t} \leq \varepsilon_{c}$ is satisfied. Set $i=1$.

6: While $i \leq N_{i}$, do

7: Train the action network

(1) Use the input-to-hidden and hidden-to-output weight vectors $s_{a}$ and $\hat{\omega}_{t}$, compute $\hat{u}_{t}$ by equation (41).

(2) Take $\hat{u}_{t}$ and $x_{t}$ into the model network to obtain the next system states $\hat{x}_{t+1}$.

(3) Compute $\hat{J}^{(i)}\left(\hat{x}_{t+1}\right)$ by equation (37).

(4) Train the action network for maximal $N_{a}$ steps by equation (43) or until $E_{a t} \leq \varepsilon_{a}$ is satisfied.

8: Train the critic network

(1) Use the input-to-hidden and hidden-to-output weight vectors $s_{c}$ and $\hat{v}_{t}$, compute $\hat{J}^{(i+1)}\left(x_{t}\right)$ and $J^{(i+1)}\left(x_{t}\right)$ by equations (36) and (38), respectively.

(2) Obtain the error function $E_{c t}$ by equation (39).

(3) Train the critic network via weight updating rule (40) for maximal $N_{c}$ steps or until $E_{c t} \leq \varepsilon_{c}$ is satisfied.

9: If $\left\|\hat{J}^{(i+1)}\left(x_{t}\right)-\hat{J}^{(i)}\left(x_{t}\right)\right\| \leq \varepsilon$, end While and obtain the approximate optimal controller; else, $i=i+1$, go to step 6.

estimation errors $\tilde{v}_{t}$ and $\tilde{\omega}_{t}$ under some reasonable assumptions.

Assumption 2 The optimal weight vectors $\omega$ and $v$ are bounded, separately for action and critic networks, such 
that $\|\omega\| \leq \omega_{M},\|v\| \leq v_{M}$, where $\omega_{M}, v_{M} \in \mathbb{R}$ are positive constants.

Assumption 3 The activation functions $\vartheta_{c t}$ and $\vartheta_{\text {at }}$ as well as the reconstruction errors $\zeta_{c t}$ are bounded, i.e., $\left\|\vartheta_{c t}\right\| \leq \vartheta_{c M},\left\|\vartheta_{a t}\right\| \leq \vartheta_{a M}$, and $\left\|\zeta_{c t}\right\| \leq \zeta_{c M}$, where $\vartheta_{c M}, \vartheta_{a M}$ and $\zeta_{c M}$ are positive constants.

Theorem 4 Considering the controlled system in equation (1) with unknown system dynamics, if all system states $x_{t}$ and control inputs $u_{t}$ are measurable, then the INDP approximate optimal controller can be obtained by Algorithm 2, the weight vectors of critic and action neural networks are regulated by equations (40) and (43), respectively. The associated weight estimation errors $\tilde{v}_{t}$ and $\tilde{\omega}_{t}$ are uniformly ultimately bounded by Assumptions 2 and 3.

PROOF. As defined above, the weight estimation errors are $\tilde{v}_{t}=\hat{v}_{t}-v$ and $\tilde{\omega}_{t}=\hat{\omega}_{t}-\omega$ for critic and action networks, respectively. The selected Lyapunov function is denoted as

$$
L(t)=\frac{1}{\eta_{c}} \operatorname{tr}\left\{\tilde{v}_{t}^{T} \tilde{v}_{t}\right\}+\frac{1}{\eta_{a}} \operatorname{tr}\left\{\tilde{\omega}_{t}^{T} \tilde{\omega}_{t}\right\},
$$

where $L_{2}(t)=\eta_{c}^{-1} \operatorname{tr}\left\{\tilde{v}_{t}^{T} \tilde{v}_{t}\right\}$ and $L_{3}(t)=\eta_{a}^{-1} \operatorname{tr}\left\{\tilde{\omega}_{t}^{T} \tilde{\omega}_{t}\right\}$. Firstly, we investigate the difference of $L_{2}(t)$, which is formulated as

$$
\begin{aligned}
\Delta L_{2}(t)= & L_{2}(t+1)-L_{2}(t) \\
= & \eta_{c}^{-1} \operatorname{tr}\left\{\tilde{v}_{t+1}^{T} \tilde{v}_{t+1}-\tilde{v}_{t}^{T} \tilde{v}_{t}\right\} \\
= & \eta_{c}^{-1} \operatorname{tr}\left\{\left(\hat{v}_{t+1}-v\right)^{T}\left(\hat{v}_{t+1}-v\right)-\tilde{v}_{t}^{T} \tilde{v}_{t}\right\} \\
= & \eta_{c}^{-1} \operatorname{tr}\left\{( \hat { v } _ { t } ^ { T } - \eta _ { c } e _ { c t } \vartheta _ { c t } ^ { T } - v ^ { T } ) \left(\hat{v}_{t}-\eta_{c} e_{c t} \vartheta_{c t}\right.\right. \\
& \left.-v)-\tilde{v}_{t}^{T} \tilde{v}_{t}\right\} \\
= & \eta_{c}^{-1} \operatorname{tr}\left\{\left(\tilde{v}_{t}^{T}-\eta_{c} e_{c t} \vartheta_{c t}^{T}\right)\left(\tilde{v}_{t}-\eta_{c} e_{c t} \vartheta_{c t}\right)\right. \\
& \left.-\tilde{v}_{t}^{T} \tilde{v}_{t}\right\} \\
= & \operatorname{tr}\left\{\eta_{c} e_{c t}^{2} \vartheta_{c t}^{T} \vartheta_{c t}-2 e_{c t} \tilde{v}_{t}^{T} \vartheta_{c t}\right\} .
\end{aligned}
$$

Considering equations (36) and (48), $e_{c t}=\hat{J}^{(i+1)}\left(x_{t}\right)-$ $J^{(i+1)}\left(x_{t}\right)=\tilde{v}_{t}^{T} \vartheta_{c t}-\zeta_{c t}$. Let $\xi_{c t}=\tilde{v}_{t}^{T} \vartheta_{c t}$. By applying the Cauchy-Schwarz inequality in Lemma 4, then we derive $\Delta L_{2}(t)$ as

$$
\begin{aligned}
\Delta L_{2}(t)= & \operatorname{tr}\left\{\eta_{c}\left(\vartheta_{c t} \xi_{c t}-\vartheta_{c t} \zeta_{c t}\right)^{T}\left(\vartheta_{c t} \xi_{c t}-\vartheta_{c t} \zeta_{c t}\right)\right. \\
& \left.-2 \xi_{c t}\left(\xi_{c t}-\zeta_{c t}\right)\right\} \\
\leq & 2 \eta_{c}\left(\left(\vartheta_{c t} \xi_{c t}\right)^{T} \vartheta_{c t} \xi_{c t}+\left(\vartheta_{c t} \zeta_{c t}\right)^{T} \vartheta_{c t} \zeta_{c t}\right) \\
& -2\left\|\xi_{c t}\right\|^{2}+2 \xi_{c t} \zeta_{c t} \\
\leq & 2 \eta_{c}\left\|\vartheta_{c t}\right\|^{2}\left(\left\|\xi_{c t}\right\|^{2}+\left\|\zeta_{c t}\right\|^{2}\right)-\left\|\xi_{c t}\right\|^{2}+\left\|\zeta_{c t}\right\|^{2} \\
= & -\left(1-2 \eta_{c}\left\|\vartheta_{c t}\right\|^{2}\right)\left\|\xi_{c t}\right\|^{2}+\left(1+2 \eta_{c}\left\|\vartheta_{c t}\right\|^{2}\right) \times \\
& \left\|\zeta_{c t}\right\|^{2} .
\end{aligned}
$$

Secondly, we consider the difference of $L_{3}(t)$, which can be obtained as

$$
\Delta L_{3}(t)=\frac{1}{\eta_{a}} \operatorname{tr}\left\{\tilde{\omega}_{t+1}^{T} \tilde{\omega}_{t+1}-\tilde{\omega}_{t}^{T} \tilde{\omega}_{t}\right\} .
$$

With the updating rule of $\hat{\omega}_{t+1}$, it gets

$$
\begin{aligned}
\tilde{\omega}_{t+1} & =\hat{\omega}_{t+1}-\omega \\
& =\tilde{\omega}_{t}-\eta_{a} \vartheta_{a t} \hat{v}_{t}^{T} \vartheta_{c}\left(z_{t+1}\right) \hat{v}_{t}^{T} \varphi_{t} \pi .
\end{aligned}
$$

Substituting the above equation into (52) and using the trace property in Lemma 3, we get

$$
\begin{aligned}
\Delta L_{3}(t)= & \frac{1}{\eta_{a}} \operatorname{tr}\left\{\left(\tilde{\omega}_{t}-\eta_{a} \vartheta_{a t} \hat{v}_{t}^{T} \vartheta_{c}\left(z_{t+1}\right) \hat{v}_{t}^{T} \varphi_{t} \pi\right)^{T} \times\right. \\
& \left.\left(\tilde{\omega}_{t}-\eta_{a} \vartheta_{a t} \hat{v}_{t}^{T} \vartheta_{c}\left(z_{t+1}\right) \hat{v}_{t}^{T} \varphi_{t} \pi\right)-\tilde{\omega}_{t}^{T} \tilde{\omega}_{t}\right\} \\
= & \operatorname{tr}\left\{-2 \tilde{\omega}_{t}^{T} \vartheta_{a t} \hat{v}_{t}^{T} \vartheta_{c}\left(z_{t+1}\right) \hat{v}_{t}^{T} \varphi_{t} \pi+\eta_{a} \pi^{T} \varphi_{t}^{T} \times\right. \\
& \left.\hat{v}_{t} \vartheta_{c}^{T}\left(z_{t+1}\right) \hat{v}_{t} \vartheta_{a t}^{T} \vartheta_{a t} \hat{v}_{t}^{T} \vartheta_{c}\left(z_{t+1}\right) \hat{v}_{t}^{T} \varphi_{t} \pi\right\} . \quad(53)
\end{aligned}
$$

Define $\xi_{a t}=\tilde{\omega}_{t}^{T} \vartheta_{a t}$, then equation (53) can be be simplified as

$$
\begin{gathered}
\Delta L_{3}(t)=\operatorname{tr}\left\{-2 \hat{v}_{t}^{T} \varphi_{t} \pi \xi_{a t} \hat{v}_{t}^{T} \vartheta_{c}\left(z_{t+1}\right)+\eta_{a} \pi^{T} \varphi_{t}^{T} \hat{v}_{t}\right. \\
\left.\vartheta_{c}^{T}\left(z_{t+1}\right) \hat{v}_{t} \vartheta_{a t}^{T} \vartheta_{a t} \hat{v}_{t}^{T} \vartheta_{c}\left(z_{t+1}\right) \hat{v}_{t}^{T} \varphi_{t} \pi\right\} \\
=\operatorname{tr}\left\{-\left(\pi^{T} \varphi_{t}^{T} \hat{v}_{t} \hat{v}_{t}^{T} \varphi_{t} \pi-\eta_{a} \pi^{T} \varphi_{t}^{T} \hat{v}_{t} \vartheta_{a t}^{T} \times\right.\right. \\
\left.\vartheta_{a t} \hat{v}_{t}^{T} \varphi_{t} \pi\right) \vartheta_{c}^{T}\left(z_{t+1}\right) \hat{v}_{t} \hat{v}_{t}^{T} \vartheta_{c}\left(z_{t+1}\right) \\
-\pi^{T} \varphi_{t}^{T} \hat{v}_{t} \xi_{a t} \xi_{a t}^{T} \hat{v}_{t}^{T} \varphi_{t} \pi+\left(\hat{v}_{t}^{T} \vartheta_{c}\left(z_{t+1}\right)\right. \\
\left.-\hat{v}_{t}^{T} \varphi_{t} \pi \xi_{a t}\right)^{T}\left(\hat{v}_{t}^{T} \vartheta_{c}\left(z_{t+1}\right)-\hat{v}_{t}^{T} \varphi_{t} \pi \xi_{a t}\right) \\
+\hat{v}_{t}^{T} \vartheta_{c}\left(z_{t+1}\right) \vartheta_{c}^{T}\left(z_{t+1}\right) \hat{v}_{t} \hat{v}_{t}^{T} \varphi_{t} \pi \pi^{T} \varphi_{t}^{T} \hat{v}_{t} \\
\left.-\vartheta_{c}^{T}\left(z_{t+1}\right) \hat{v}_{t} \hat{v}_{t}^{T} \vartheta_{c}\left(z_{t+1}\right)\right\} .
\end{gathered}
$$

Considering $\vartheta_{a t} \in \mathbb{R}^{h_{a}}, \xi_{a t} \in \mathbb{R}^{m}, \hat{v}_{t}^{T} \vartheta_{c}\left(z_{t+1}\right) \in \mathbb{R}$ and $\hat{v}_{t}^{T} \varphi_{t} \pi \in \mathbb{R}^{1 \times m}$, the following inequality can be derived from equation (54), which is

$$
\begin{aligned}
\Delta L_{3}(t)= & -\left(\left\|\hat{v}_{t}^{T} \varphi_{t} \pi\right\|^{2}-\eta_{a}\left\|\hat{v}_{t}^{T} \varphi_{t} \pi\right\|^{2}\left\|\vartheta_{a t}\right\|^{2}\right) \\
& \times\left\|\hat{v}_{t}^{T} \vartheta_{c}\left(z_{t+1}\right)\right\|^{2}-\left\|\hat{v}_{t}^{T} \varphi_{t} \pi \xi_{a t}\right\|^{2}+\| \hat{v}_{t}^{T} \\
& \times \vartheta_{c}\left(z_{t+1}\right)-\hat{v}_{t}^{T} \varphi_{t} \pi \xi_{a t}\left\|^{2}+\right\| \hat{v}_{t}^{T} \vartheta_{c}\left(z_{t+1}\right) \|^{2} \\
& \times\left\|\hat{v}_{t}^{T} \varphi_{t} \pi\right\|^{2}-\left\|\hat{v}_{t}^{T} \vartheta_{c}\left(z_{t+1}\right)\right\|^{2}
\end{aligned}
$$

Using the Cauchy-Schwarz inequality in Lemma 4, equa- 
tion (55) can be deduced as

$$
\begin{aligned}
\Delta L_{3}(t) \leq & -\left(\left\|\hat{v}_{t}^{T} \varphi_{t} \pi\right\|^{2}-\eta_{a}\left\|\hat{v}_{t}^{T} \varphi_{t} \pi\right\|^{2}\left\|\vartheta_{a t}\right\|^{2}\right) \times \\
& \left\|\hat{v}_{t}^{T} \vartheta_{c}\left(z_{t+1}\right)\right\|^{2}-\left\|\hat{v}_{t}^{T} \varphi_{t} \pi \xi_{a t}\right\|^{2}+2 \| \hat{v}_{t}^{T} \times \\
& \vartheta_{c}\left(z_{t+1}\right)\left\|^{2}+2\right\| \hat{v}_{t}^{T} \varphi_{t} \pi \xi_{a t}\left\|^{2}+\frac{1}{2}\right\| \hat{v}_{t}^{T} \times \\
& \vartheta_{c}\left(z_{t+1}\right)\left\|^{4}+\frac{1}{2}\right\| \hat{v}_{t}^{T} \varphi_{t} \pi\left\|^{4}-\right\| \hat{v}_{t}^{T} \vartheta_{c}\left(z_{t+1}\right) \|^{2} \\
= & -\left(\left\|\hat{v}_{t}^{T} \varphi_{t} \pi\right\|^{2}-\eta_{a}\left\|\hat{v}_{t}^{T} \varphi_{t} \pi\right\|^{2}\left\|\vartheta_{a t}\right\|^{2}\right) \times \\
& \left\|\hat{v}_{t}^{T} \vartheta_{c}\left(z_{t+1}\right)\right\|^{2}+\frac{1}{2}\left\|\xi_{a t}\right\|^{4}+\left\|\hat{v}_{t}^{T} \vartheta_{c}\left(z_{t+1}\right)\right\|^{2} \\
& +\frac{1}{2}\left\|\hat{v}_{t}^{T} \vartheta_{c}\left(z_{t+1}\right)\right\|^{4}+\left\|\hat{v}_{t}^{T} \varphi_{t} \pi\right\|^{4} .
\end{aligned}
$$

Based on equations (51) and (56), we can conclude that the difference of $L(t)$ satisfies

$$
\begin{array}{r}
\Delta L(t) \leq-\left(1-2 \eta_{c}\left\|\vartheta_{c t}\right\|^{2}\right)\left\|\xi_{c t}\right\|^{2}-\left(\left\|\hat{v}_{t}^{T} \varphi_{t} \pi\right\|^{2}-\eta_{a} \times\right. \\
\left.\left\|\hat{v}_{t}^{T} \varphi_{t} \pi\right\|^{2}\left\|\vartheta_{a t}\right\|^{2}\right)\left\|\hat{v}_{t}^{T} \vartheta_{c}\left(z_{t+1}\right)\right\|^{2}+D^{2}, \quad \text { (57) }
\end{array}
$$

where $D^{2}$ defines as

$$
\begin{aligned}
D^{2}= & \left(1+2 \eta_{c}\left\|\vartheta_{c t}\right\|^{2}\right)\left\|\zeta_{c t}\right\|^{2}+\left\|\hat{v}_{t}^{T} \vartheta_{c}\left(z_{t+1}\right)\right\|^{2} \\
& +\frac{1}{2}\left\|\xi_{a t}\right\|^{4}+\frac{1}{2}\left\|\hat{v}_{t}^{T} \vartheta_{c}\left(z_{t+1}\right)\right\|^{4}+\left\|\hat{v}_{t}^{T} \varphi_{t} \pi\right\|^{4} \\
\leq & \left(1+2 \eta_{c} \vartheta_{c M}^{2}\right) \zeta_{c M}+\left(v_{M}^{T} \vartheta_{c M}\right)^{2}+\frac{1}{2}\left(\left(\omega_{M}^{T} \vartheta_{a M}\right)^{4}\right. \\
& \left.+\left(v_{M}^{T} \vartheta_{c M}\right)^{4}+2\left(v_{M}^{T} \varphi_{M} \pi\right)^{4}\right) \\
= & D_{M}^{2}
\end{aligned}
$$

where $v_{M}, \vartheta_{c M}, \zeta_{c M}, \varphi_{M}, \omega_{M}$ and $\vartheta_{a M}$ are the upper bounds of $v_{t}, \vartheta_{c t}, \zeta_{c t}, \varphi_{t}, \omega_{t}$ and $\vartheta_{a t}$, respectively. Therefore, if

$0<\eta_{c}<\frac{1}{2 \vartheta_{c M}^{2}}, 0<\eta_{a}<\frac{1}{\vartheta_{a M}^{2}},\left\|\xi_{c t}\right\|>\sqrt{\frac{D_{M}^{2}}{1-2 \eta_{c} \vartheta_{c M}^{2}}}$,

then the first difference $\Delta L(t) \leq 0$ holds according to inequality (56). Based on the Lyapunov extension theorem, this demonstrates that $\tilde{v}_{t}$ and $\tilde{\omega}_{t}$ are uniformly ultimately bounded, which thus completes the proof.

\section{Simulation studies}

In this section, the proposed INDP algorithm has been applied on three representative examples. There is no prior knowledge about the controlled plants but only system data in our method. Simulations are presented to show the implementation and the control performance of this proposed algorithm.

Case 1 Consider the discrete-time affine nonlinear system:

$$
x_{t+1}=\left[\begin{array}{c}
x_{1 t} x_{2 t} \\
x_{1 t}^{2}-0.5 \sin \left(x_{2 t}\right) \\
x_{3 t}
\end{array}\right]+\left[\begin{array}{c}
0 \\
1 \\
-1
\end{array}\right] u_{t},
$$

where $x_{t}=\left[x_{1 t}, x_{2 t}, x_{3 t}\right]^{T} \in \mathbb{R}^{3}$ and $u_{t} \in \mathbb{R}$ are the state vector and the control variable, respectively, $x_{0}=[-0.5,0.5,1]^{T}$. The utility function is chosen as the quadratic form $R\left(x_{t}, u_{t}\right)=x_{t}^{T} P x_{t}+u_{t}^{T} Q u_{t}$, where $P$ and $Q$ are both identity matrices with appropriate dimensions.

Three-layer feedforward neural networks are used to construct the model network, the critic network, and the action network with structures $4-8-3,3-8-1$, and $3-8-1$, respectively. The INDP algorithm is applied at time step $t=0$. The input-to-hidden weight vectors of the three networks are randomly set in $[-0.5,0.5]$ and then is kept unchanged, and the hidden-to-output weight vectors are randomly initialized in $[-0.5,0.5] .1000$ measured data samples are used to train the model neural network under the learning rate $\eta_{m}=0.1$. Once the training process is completed, the hidden-to-output weight vector is also kept unchanged in the subsequent programming. Based on the uniformly ultimately bounded stability, the used parameter values of the INDP algorithm are listed in Table 1, and the meaning of the parameters can refer to Algorithm 2.

Table 1

Used parameter values in the INDP algorithm

\begin{tabular}{ll|ll|ll|ll}
\hline para. & value & para. & value & para. & value & para. & value \\
\hline$\beta$ & 1 & $\eta_{m}$ & 0.1 & $\eta_{a}$ & 0.1 & $\eta_{c}$ & 0.1 \\
$N_{i}$ & 200 & $N_{m}$ & 2000 & $N_{a}$ & 2000 & $N_{c}$ & 2000 \\
$\varepsilon$ & $10^{-6}$ & $\varepsilon_{m}$ & $10^{-6}$ & $\varepsilon_{a}$ & $10^{-12}$ & $\varepsilon_{c}$ & $10^{-12}$ \\
\hline
\end{tabular}
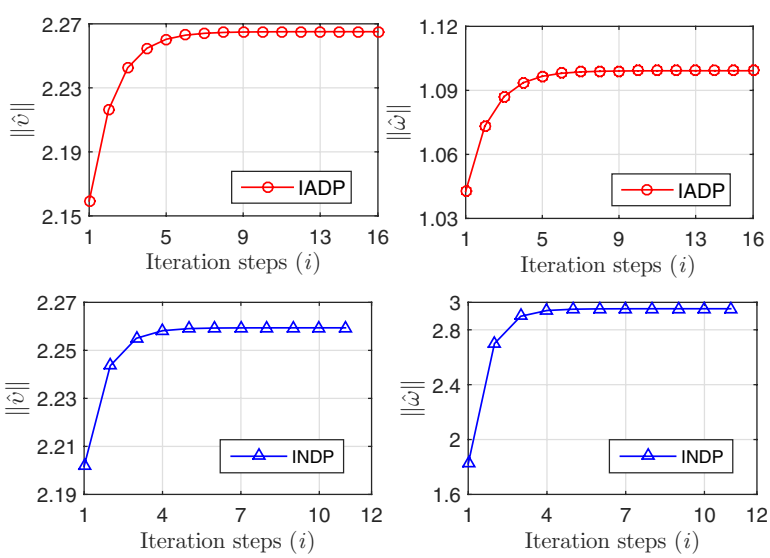

Fig. 2. The norm of weight vectors for action and critic networks with INDP and IADP methods. 


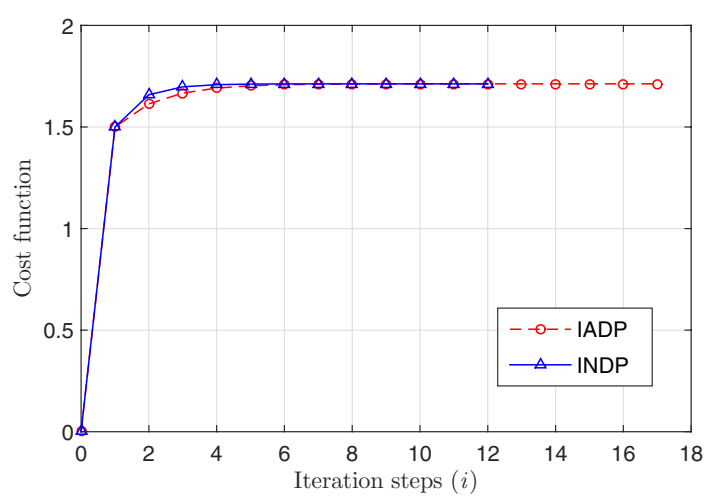

Fig. 3. The convergence curves of cost functions with INDP and IADP methods.

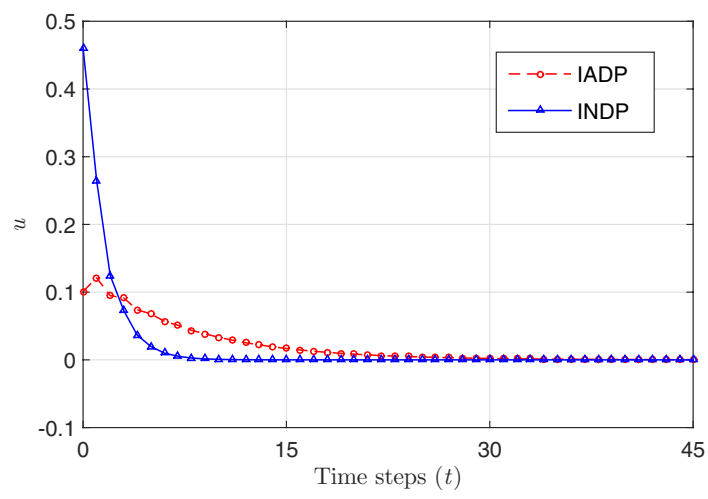

Fig. 4. The control curves of INDP and IADP methods.

With these parameters, we adapt the INDP method to stabilize the controlled system. The critic network and the action network are trained for 11 iterations (i.e., for $i=1,2, \ldots, 11)$ with 2000 inner-loop training steps for each iteration to make sure the tolerant error $\varepsilon=10^{-6}$ can be reached. In order to facilitate the comparison with the IADP method, we set the same settings on parameter values and the structures of neural networks for the two methods. As a result, the IADP method carries out 16 iterations with 2000 inner-loop training steps in each iteration to reach the tolerant error $\varepsilon=10^{-6}$.
During training processes, the norm of weight vectors for critic and action networks together with the iterative cost function, are all convergent, as shown in Figs. 2 and 3. It can be observed that the two iterative cost function sequences converge in a monotonically increasing way to the optimal cost function, which coincides with Theorem 1 and indicates the validity of the INDP algorithm only based on system data.

After training, the approximate optimal control laws based on IADP and INDP methods are derived from the trained weight vectors. Then the derived control laws are applied to the controlled system for 45 time steps to present control performance. The control curves are presented in Fig. 4. The system states are stabilized to zero under this control, as illustrated in Fig. 5. During the regulation, the curves of utility functions are shown in Fig. 6. The simulation results demonstrate that the approximate optimal control law derived by the INDP algorithm is effective only based on measured system data, and it is able to provide the excellent control performance.

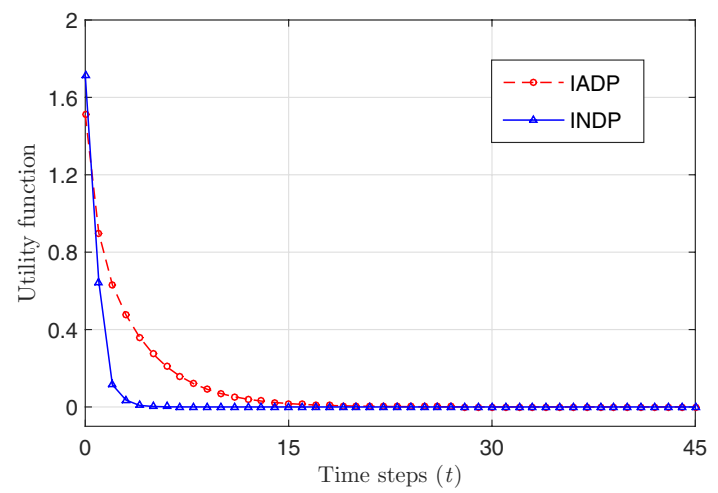

Fig. 6. The values of utility functions with INDP and IADP methods.

Case 2 In this example, we consider the following non-

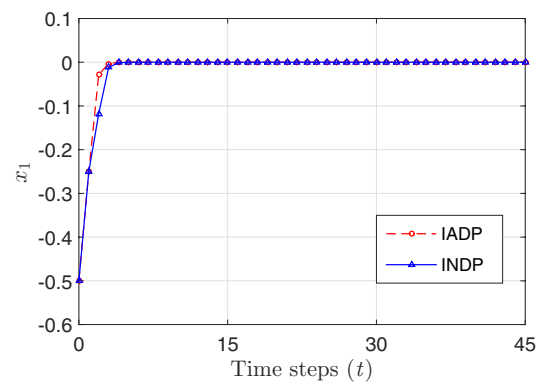

(a)

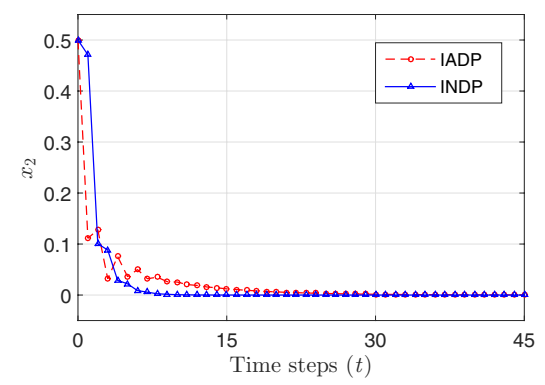

(b)

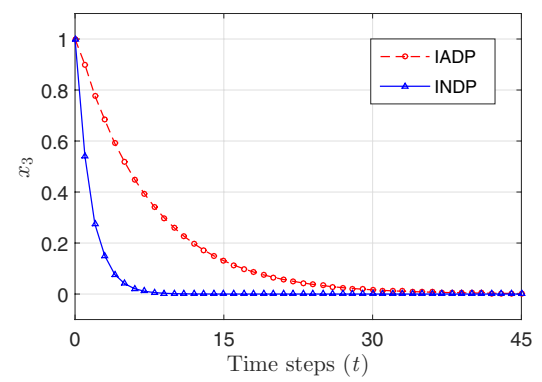

(c)

Fig. 5. The control performance with INDP and IADP methods, (a) the trajectories of $x_{1}$, (b) the trajectories of $x_{2}$, (c) the trajectories of $x_{3}$. 
affine discrete-time nonlinear system:

$$
x_{t+1}=1.2 x_{t}+\sin \left(0.8 x_{t}^{2}+\tanh \left(u_{t}\right)\right),
$$

where $x_{t}$ and $u_{t}$ are the state vector and the control variable, respectively, $x_{0}=0.6$. The utility function is chosen the same formulation as in case 1.

Since the control variable cannot be explicitly expressed in $x_{t+1}$, it is very difficult to apply the IADP method which needs an explicit control matrix $g_{t}$ or its estimation $\hat{g}_{t}$. Even if the model network is used for this kind of nonlinear system, it is obviously inaccurate because the explicit relation between $u_{t}$ and $x_{t+1}$ cannot be guaranteed. The proposed INDP method has the ability to deal with this kind of nonlinear systems.

The model, critic and action networks are chosen with structures 2-6-1, 1-6-1 and 1-6-1, respectively. All the weight vectors of three networks are randomly initialized in $[-0.1,0.1]$, then the input-to-hidden weight vectors are kept unchanged, and only the hidden-to-output weight vectors are updated. All used parameters can refer to Table 1. Before starting the INDP algorithm, we first train the model network by using 1000 measured system data samples derived from the non-affine nonlinear system, and then remain its weight vector during the whole implementation of the INDP algorithm.

The INDP algorithm starts from $i=0$ and $t=0$ with an initial iterative cost function $J^{(0)}(\cdot)=0$, the associated iterative control input $u^{(0)}(\cdot)=0$ and the initial state $x_{0}=0.6$. Let $i=1,2, \cdots, N_{i}$, using the input-to-hidden weight vector $s_{c}$ and hidden-to-output weight vector $\hat{v}_{t}$, $\hat{J}^{(i+1)}\left(x_{t}\right)$ and $J^{(i+1)}\left(x_{t}\right)$ can be calculated by equations (36) and (38), respectively. $\hat{v}_{t}$ is updated via 2000 innerloop steps by equation (40) on basis of the error function $E_{c t}$. The updating process of weights for the critic network is shown in Fig. 7, where Fig. 7(a) provides weight convergence curves as the iteration steps increase and Fig. 7 (b) specifically presents that the weights are updated in the inner loops by minimizing the error function of critic network. Meanwhile, the control input $\hat{u}_{t}$ is calculated by the action network with the weight vectors $s_{a}$ and $\hat{\omega}_{t} . \hat{u}_{t}$ and $x_{t}$ are transmitted into the model network to produce $\hat{x}_{t+1} . \hat{J}^{(i)}\left(\hat{x}_{t+1}\right)$ is calculated by equation (37). Then $\hat{\omega}_{t}$ in the action network can be updated by employing equation (43) via 2000 inner-loop steps based on $E_{a t}$. Similarly, the weights regulation are presented in Fig. 8, where Fig. 8 (a) illustrates the weights are updated as the iteration steps increase and Fig. 8 (b) depicts the detailed weight updating in the inner loops to minimize the error function of action network. We check the condition $\left\|\hat{J}^{(i+1)}\left(x_{t}\right)-\hat{J}^{(i)}\left(x_{t}\right)\right\| \leq \varepsilon$ to examine whether it can be satisfied or not. When $i=18$, this condition holds and the convergence curve of iterative cost function is shown in Fig. 9 (a). Therefore we obtain the approximated optimal control law with $\hat{v}_{t}$ and

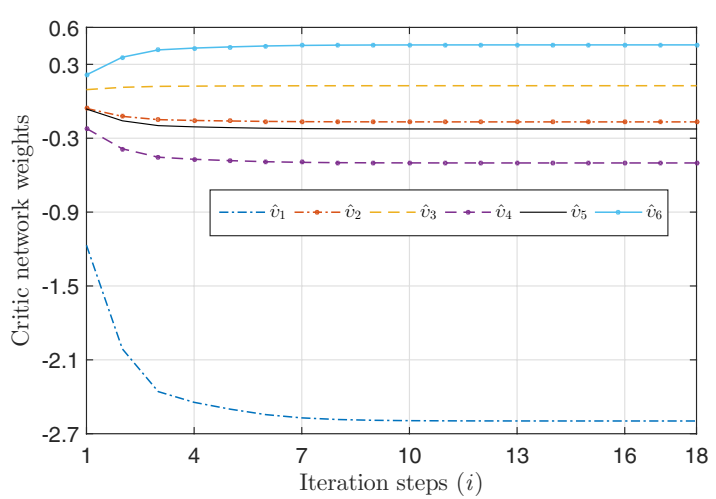

(a)

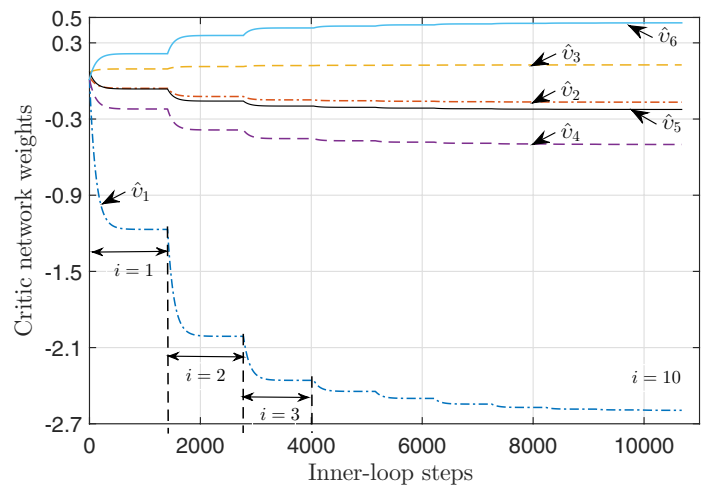

(b)

Fig. 7. The updating process of weights for the critic network, (a) the weight updating with the iteration steps increasing, (b) the weight updating with both inner-loop steps and iteration steps increasing.

$\hat{\omega}_{t}$ and apply it to control the non-affine nonlinear system for 30 time steps. The state curve is in Fig. 9 (b) and the control curve is in Fig. 9 (c), where the state of the non-affine nonlinear system is stabilized to zero. It verifies the effectiveness of this proposed method to deal with the control problem of this kind nonlinear systems.

Case 3 In this case, we apply the proposed INDP algorithm to the dynamical system of single-link manipulator, whose mechanical model is shown in Fig. 10, which is also discussed in (Wu and Cai, 2006; Zhong, Ni and $\mathrm{He}, 2016)$ The mathematical description of this system is formulated as the following second-order differential equation:

$$
\left\{\begin{array}{l}
\frac{\mathrm{d} \theta}{\mathrm{d} t}=w, \\
\frac{\mathrm{d} w}{\mathrm{~d} t}=G^{-1}\left(u_{t}-M g L \sin (\theta)-f_{d} \frac{\mathrm{d} \theta}{\mathrm{d} t}\right),
\end{array}\right.
$$

where $M=1 \mathrm{~kg}$ is the mass of manipulator, and $L=0.5$ $\mathrm{m}$ is the length of manipulator. The system states are the current angle $\theta$ and the associated angular velocity $w$. Let $G=5 \mathrm{~kg} \cdot \mathrm{m}^{2}, f_{d}=2 \mathrm{~kg} \cdot \mathrm{m}^{2}$ and $g=9.81 \mathrm{~m} / \mathrm{s}^{2}$ be the moment of inertia, the viscous friction and the 


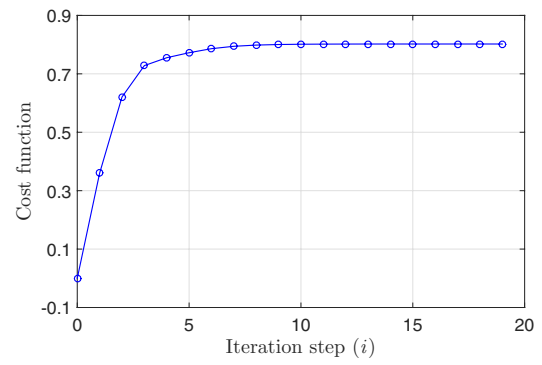

(a)

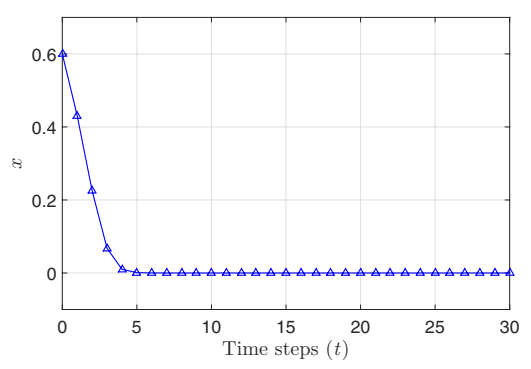

(b)

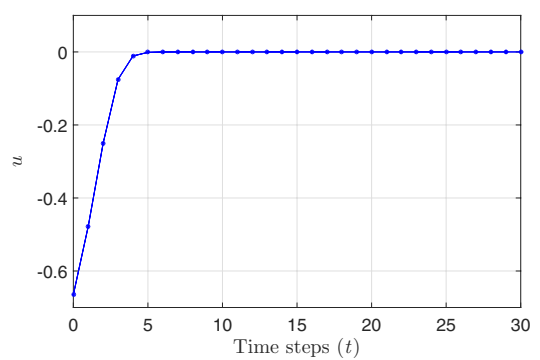

(c)

Fig. 9. The control performance of INDP algorithm for case 2, (a) the convergence curve of the iterative cost function, (b) the trajectory of the state, (c) the control input.

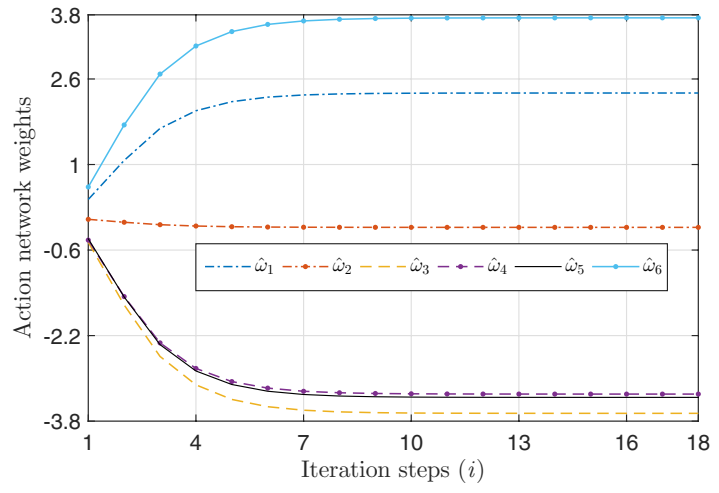

(a)

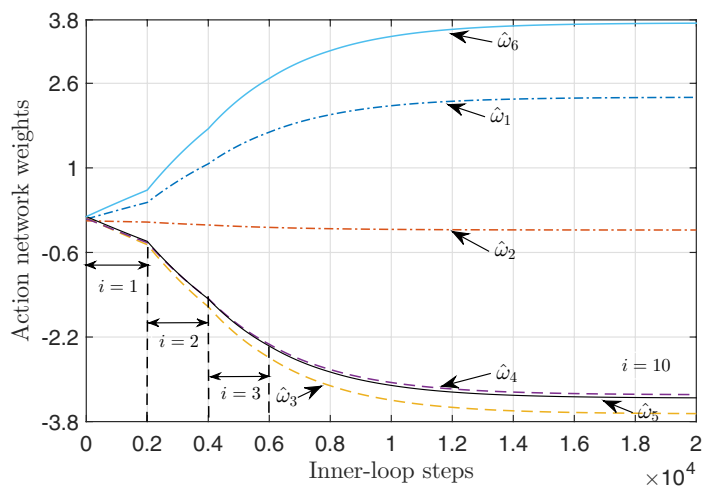

(b)

Fig. 8. The updating process of weights for the action network, (a) the weight updating with the iteration steps increasing, (b) the weight updating with both inner-loop steps and iteration steps increasing.

acceleration of gravity, respectively.

The Euler method with the sampling interval $\Delta t=0.1 \mathrm{~s}$ is used to discretize this system as (Chapra and Canale, 1998; Wei, Lewis, Liu, Song and Lin, 2016)

$x_{t+1}=\left[\begin{array}{c}x_{1 t}+0.1 x_{2 t} \\ \frac{1}{5}\left(-0.49 \sin \left(x_{1 t}\right)-0.2 x_{2 t}+x_{2 t}\right)\end{array}\right]+\left[\begin{array}{c}0 \\ \frac{0.1}{5}\end{array}\right] u_{t}$,
Fig. 10. The mechanical model of the single-link manipulator

where $x_{1}=\theta$ and $x_{2}=w$. The initial state vector of the controlled system is $x_{0}=[-1,1]^{T}$. The model network, the critic network and the action network are with the structures of $3-8-2,2-8-1$ and $2-8-1$. The tolerant error of INDP algorithm is set as $\varepsilon=10^{-4}$. All other parameters are kept the same settings as in Table 1.

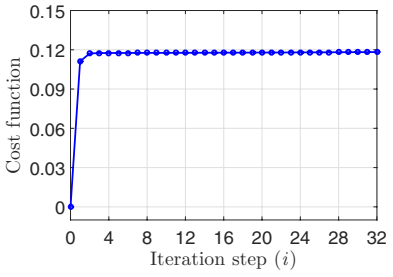

(a)

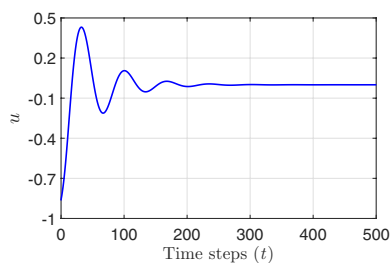

(c)

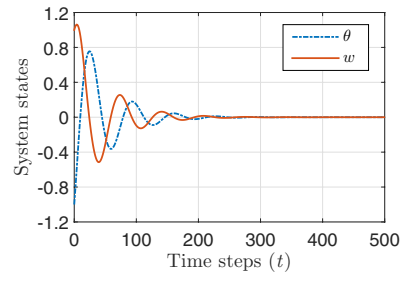

(b)

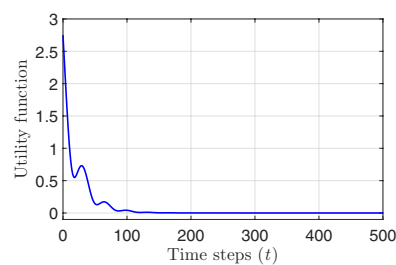

(d)
Fig. 11. The control performance of the INDP method for case 3, (a) the convergence curve of the iterative cost function, (b) the trajectories of the states, (c) the control curve, (d) the utility function.

The model network are first sufficiently trained based on 1000 data samples, then the trained $\hat{\nu}_{t}$ and the given $s_{m}$ are fixed for the INDP algorithm. We then train 
the critic network and the action network for maximal 200 iteration steps with 2000 internal loops for each iteration. When $i=31$, the iterative cost function $\hat{J}^{(31)}=0.1181713$ and $\hat{J}^{(32)}=0.1182044$, it means that $\left\|\hat{J}^{(i+1)}-\hat{J}^{(i)}\right\| \leq \varepsilon$ holds, and the tolerance error $\varepsilon=10^{-4}$ is achieved when $i=31$. Fig. 11 (a) shows the convergence curve of the iterative cost function. After that, the obtained approximate optimal control law is applied to the single-link manipulator for 500 time steps. Fig. 11 (b) presents two state trajectories of angle $\theta$ and angular velocity $w$. Fig. 11 (c) gives the control curve and Fig. 11 (d) provides the utility function during the control process. Fig. 12 illustrates the phase trajectory of the single-link manipulator under the INDP controller over time. All control performance including the state trajectories, the phase trajectory, the control curve and the utility curve demonstrates that the INDP controller can provide the effective approximate optimal control law to make the manipulator stay in the origin after a transient regulation, while the associated control law is produced only based on all measurable system data.

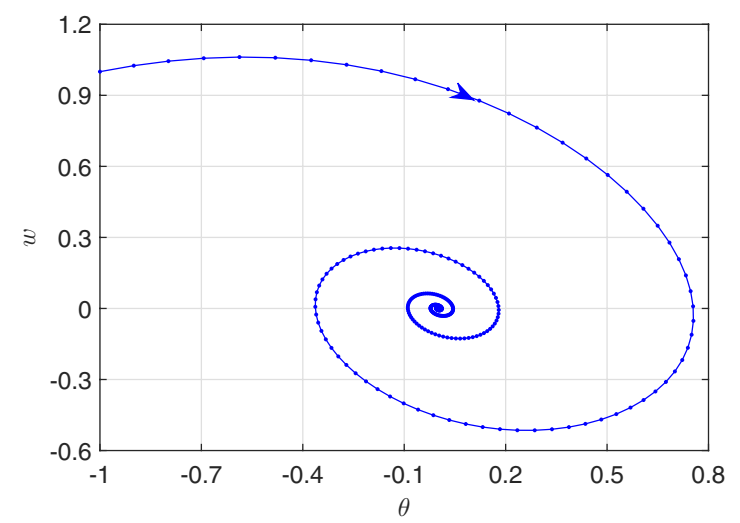

Fig. 12. The phase trajectory under the INDP controller for the single-link manipulator

\section{Conclusion}

In this paper, an effective INDP algorithm is proposed to obtain the approximate optimal controller for unknown affine and non-affine nonlinear discrete-time systems. The INDP algorithm solves the discrete-time HJB equation within the framework of IADP and its implementation is dependent on measured system data without inquiring the system mathematic model. The iteration convergence analysis and the uniformly ultimately boundness for the algorithm implementation are both provided. Three example studies are presented to show the control performance derived from the proposed INDP algorithm, which illustrate that the proposed scheme is a data-driven control strategy and it can stabilize general discrete-time nonlinear system by learning from system data. For further research work, the INDP approach based on a DHP/GDHP structure can be developed with the associated stability analysis. Also, we will explore this INDP approach to uncertain systems and constrained systems.

\section{References}

Abu-Khalaf, M. and Lewis, F. L. (2004), 'Nearly optimal state feedback control of constrained nonlinear systems using a neural networks HJB approach', Annual Reviews in Control 28(2), 239-251.

Al-Tamimi, A., Lewis, F. L. and Abu-Khalaf, M. (2008), 'Discrete-time nonlinear HJB solution using approximate dynamic programming: convergence proof', IEEE Transactions on System, Man, Cybernetics, Part B: Cybernetics 38, 943-949.

Beard, R. W., Saridis, G. N. and Wen, J. T. (1997), 'Galerkin approximations of the generalized Hamilton-Jacobi-Bellman equation', Automatica 33(12), 2159-2177.

Bellman, R. (1957), Dynamic Programming, Princeton University Press, Princeton, NJ, USA.

Chapra, S. C. and Canale, R. P. (1998), Numerical Methods For Engineers, McGraw-Hill.

Dierks, T., Thumati, B. T. and Jagannathan, S. (2009), 'Optimal control of unknown affine nonlinear discretetime systems using offline-trained neural networks with proof of convergence', Neural Networks 22(56), 851-860.

Fan, Q. and Yang, G. (2016), 'Adaptive actor-critic design-based integral sliding-mode control for partially unknown nonlinear systems with input disturbances', IEEE Transactions on Neural Networks and Learning Systems 27(1), 165-177.

He, H., Ni, Z. and Fu, J. (2012), 'A three-network architecture for on-line learning and optimization based on adaptive dynamic programming', Neurocomputing 78(1), 3-13.

Heydari, A. (2014), 'Revisiting approximate dynamic programming and its convergence', IEEE Transactions on Cybernetics 44(12), 2733-2743.

Heydari, A. (2016), 'Theoretical and numerical analysis of approximate dynamic programming with approximation errors', Journal of Guidance, Control, and Dynamics 39(2), 301-311.

Heydari, A. and Balakrishnan, S. N. (2014a), 'An adaptive critic-based scheme for consensus control of nonlinear multi-agent systems', International Journal of Control 87(12), 2463-2474.

Heydari, A. and Balakrishnan, S. N. (2014b), 'Optimal switching and control of nonlinear switching systems using approximate dynamic programming', IEEE Transactions on Neural Networks and Learning Systems 25(6), 1106-1117.

Igelnik, B. and Pao, Y.-H. (1995), 'Stochastic choice of basis functions in adaptive function approximation and the functional-link net', IEEE Transactions on Neural Networks 6(6), 1320-1329. 
Jiang, Y. and Jiang, Z.-P. (2012), 'Computational adaptive optimal control for continuous-time linear systems with completely unknown dynamics', Automatica 48(10), 2699-2704.

Jiang, Y. and Jiang, Z.-P. (2014), 'Robust adaptive dynamic programming and feedback stabilization of nonlinear systems', IEEE Transactions on Neural Networks and Learning Systems 25(5), 882-893.

Kleinman, D. (1968), 'On an iterative technique for Riccati equation computations', IEEE Transactions on Automatic Control 13(1), 114-115.

Lewis, F. L. and Syrmos, V. L. (1995), Optimal control, John Wiley \& Sons.

Luo, B., Wu, H.-N., Huang, T. and Liu, D. (2015), 'Reinforcement learning solution for HJB equation arising in constrained optimal control problem', Neural Networks 71, 150-158.

Mu, C., Ni, Z., Sun, C. and He, H. (2016a), 'Airbreathing hypersonic vehicle tracking control based on adaptive dynamic programming', IEEE Transactions on Neural Networks and Learning Systems . In press, DOI:10.1109/TNNLS.2016.2516948.

Mu, C., Ni, Z., Sun, C. and He, H. (2016b), 'Datadriven tracking control with adaptive dynamic programming for a class of continuous-time nonlinear systems', IEEE Transactions on Cybernetics . In press, DOI: $10.1109 /$ TCYB.2016.2548941.

Mu, C., Sun, C., Song, A. and Yu, H. (2016), 'Iterative GDHP-based approximate optimal tracking control for a class of discrete-time nonlinear systems', Neurocomputing 214, 775-784.

Ni, Z., He, H., Wen, J. and Xu, X. (2013), 'Goal representation heuristic dynamic programming on maze navigation', IEEE Transactions Neural Networks and Learning Systems 24(12), 2038-2050.

Prokhorov, D. V., Santiago, R. A. and Wunsch, D. C. (1995), 'Adaptive critic designs: a case study for neuro control', Neural Networks 8(9), 1367-1372.

Saridis, G. N. and Lee, C. S. G. (1979), 'An approximation theory of optimal control for trainable manipulators', IEEE Transactions on Systems, Man, and Cybernetics $\mathbf{9}(3), 152-159$.

Si, J., Barto, A. G., Powell, W. B. and Wunsch, D. (2004), Handbook of Learning and Approximate Dynamic Programming, IEEE Press/Wiley, NewYork, NY, USA.

Si, J. and Wang, Y. T. (2001), 'On-line learning control by association and reinforcement', IEEE Transactions on Neural Networks 12, 264-276.

Tang, Y., Mu, C. and He, H. (2016), 'SMES based damping controller design using fuzzy-GrHDP considering transmission delay', IEEE Transactions on Applied Superconductivity 26(7), 1-5.

Vamvoudakis, K. G. and Lewis, F. L. (2010), 'Online actor-critic algorithm to solve the continuous-time infinite horizon optimal control problem', Automatica 46(5), 878-888.

Vamvoudakis, K. G., Mojoodi, A. and Ferraz, H. (2016), 'Event-triggered optimal tracking control of nonlinear systems', International Journal of Robust and Nonlinear Control. In press, DOI: 10.1002/rnc.3587.

Wang, D. and Liu, D. (2013), 'Neuro-optimal control for a class of unknown nonlinear dynamic systems using SN-DHP technique', Neurocomputing 121, 218-225.

Wang, D., Liu, D., Wei, Q., Zhao, D. and Jin, N. (2012), 'Optimal control of unknown nonaffine nonlinear discrete-time systems based on adaptive dynamic programming', Automatica 48(8), 1825-1832.

Wang, D., Liu, D., Zhang, Q. and Zhao, D. (2016), 'Databased adaptive critic designs for nonlinear robust optimal control with uncertain dynamics', IEEE Transactions on Systems, Man, and Cybernetics: Systems 46(11), 1544-1555.

Wang, D., Mu, C., He, H. and Liu, D. (2016), 'Eventdriven adaptive robust control of nonlinear systems with uncertainties through NDP strategy', IEEE Transactions on Systems, Man, and Cybernetics: Systems. In press, DOI: 10.1109/TSMC.2016.2592682.

Wang, D., Mu, C. and Liu, D. (2017), 'Data-driven nonlinear near-optimal regulation based on iterative neural dynamic programming', Acta Automatica Sinica 43(3), 354-363.

Wang, D., Mu, C., Liu, D. and Ma, H. (2016), 'On mixed data and event driven design for adaptive-critic-based nonlinear $\mathrm{H} \infty$ control', IEEE Transactions on Neural Networks and Learning Systems. In press, DOI: 10.1109/TNNLS.2016.2642128.

Wang, F.-Y., Jin, N., Liu, D. and Wei, Q. (2011), 'Adaptive dynamic programming for finite-horizon optimal control of discrete-time nonlinear systems with $\varepsilon$ error bound', IEEE Transactions on Neural Networks 22(1), 24-36.

Wei, Q., Lewis, F. L., Liu, D., Song, R. and Lin, H. (2016), 'Discrete-time local value iteration adaptive dynamic programming: convergence analysis', IEEE Transactions on Systems, Man, and Cybernetics: Systems . In press, DOI: 10.1109/TSMC.2016.2623766.

Werbos, P. J. (1992), Approximate dynamic programming for real-time control and neural modeling, in: Handbook of Intelligent Control, Van Nostrand, NewYork, NY, USA.

Wu, H.-N. and Cai, K.-Y. (2006), 'Mode-independent robust stabilization for uncertain markovian jump nonlinear systems via fuzzy control', IEEE Transactions on Systems, Man, And Cybernetics-Part B: Cybernetics 36(3), 509-519.

Yang, L., Si, J., Tsakalis, K. S. and Rodriguez, A. A. (2009), 'Direct heuristic dynamic programming for nonlinear tracking control with filtered tracking error', IEEE Transactions on Systems, Man, and Cybernetics, Part B: Cybernetics 39(6), 1617-1622.

Yang, X., Liu, D., Wang, D. and Wei, Q. (2014), 'Discrete-time online learning control for a class of unknown nonaffine nonlinear systems using reinforcement learning', Neural Networks 55, 30-41.

Zhang, H., Cui, L., Zhang, X. and Luo, Y. (2011), 'Datadriven robust approximate optimal tracking control for unknown general nonlinear systems using adaptive 
dynamic programming method', IEEE Transactions on Neural Networks 22(12), 2226-2236.

Zhang, H., Liang, H., Wang, Z. and Feng, T. (2015), 'Optimal output regulation for heterogeneous multiagent systems via adaptive dynamic programming', IEEE Transactions on Neural Networks and Learning Systems. In press, DOI: 10.1109/TNNLS.2015.2499757.

Zhang, H., Luo, Y. and Liu, D. (2009), 'Neural-networkbased near-optimal control for a class of discrete-time affine nonlinear systems with control constraints', IEEE Transactions on Neural Networks 20, 14901503.

Zhong, X., Ni, Z. and He, H. (2016), 'A theoretical foundation of goal representation heuristic dynamic programming', IEEE Transactions on Neural Networks and Learning Systems 27(12), 2513-2525. 